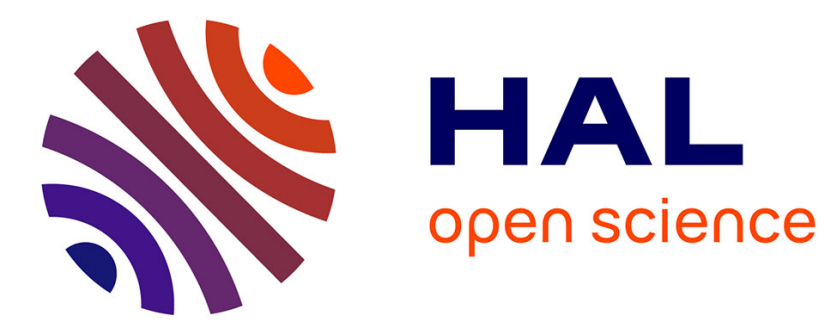

\title{
Les lits grecs en bronze de Bourgoin-Jallieu (Isère) Stéphanie Boucher
}

\section{To cite this version:}

Stéphanie Boucher. Les lits grecs en bronze de Bourgoin-Jallieu (Isère). Gallia - Fouilles et monuments archéologiques en France métropolitaine, 1982, 40 (1), pp.171-193. 10.3406/galia.1982.1858 . hal01940317

\section{HAL Id: hal-01940317 \\ https://hal.science/hal-01940317}

Submitted on 27 Feb 2020

HAL is a multi-disciplinary open access archive for the deposit and dissemination of scientific research documents, whether they are published or not. The documents may come from teaching and research institutions in France or abroad, or from public or private research centers.
L'archive ouverte pluridisciplinaire $\mathbf{H A L}$, est destinée au dépôt et à la diffusion de documents scientifiques de niveau recherche, publiés ou non, émanant des établissements d'enseignement et de recherche français ou étrangers, des laboratoires publics ou privés.

\section{(이) $\$$}

Distributed under a Creative Commons Attribution - NonCommercial - NoDerivatives| 4.0 


\title{
LES LITS GRECS EN BRONZE DE BOURGOIN-JALLIEU (Isère)
}

\author{
par Stéphanie BOUCHER
}

Le 16 mai 1863 fut donné par M. le duc de Luynes au Musée des Beaux-Arts à Lyon un ensemble d'objets décrits de la facon suivante" :Quarante-deux pièces de bronze faisant partic d'un siège antique, trouvé à Jallieu(x) près de Bourgoin il y a quinze ans, en minant un terrain, par M. Roche. Le vendeur, M. Thibaud, est au Lycée de Lyon. M. Thibaud, dit-on, possède les autres pièces de ce monument ". Nous n'avons pu retrouver trace ni de M. Roche, ni de M. Thibaud, non plus qu'aucun document concernant les objets qui étaient en possession de M. le duc de Luynes avant qu il en fît clon au Musée des BeauxArts.

Depuis, ces pièces de bronze ont été transférées au Yusée de la Civilisation galloromaine à Lyon, qui possède quatre pieds de meuble reconstilués provisoirement (fig. 1)2 comportant chacun neuf éléments superposés, dont l'un est moderne ( $b$ IV, voir le tableau p. 191), soit trente-cinq pièces, et sept éléments de deux autres pieds (fig. 2) ${ }^{3}$. Le total est donc de quarante-deux pièces, correspondant apparemment à l'inventaire d'entrée.

En 1891 cependant, II. Bazin mentionne un bisellium non remonté, incrusté d'argent, et en 1899, un catalogue sommaire sans nom d'auteur, mais rédige probablement sous la direction de P. Dissarl, alors conservateur, fait état également de ce bisellium, donné au Musée par M. le duc de Luynes, el incrusté d’argent. Or une seule des quarante-decux pièces que nous possédons, un sorle de pied (a VIII) présente des incisions en creux, en forme de rectangles et de grecques qui, peut-être, ont été incrustées d'argent (fig. 3), n'en conservent aucune trace ot ne justifient nullement des mentions aussi laudatives. lin 1905, (:. L. Ransom ${ }^{6}$ publie le meuble en question, ef sur les photographies qui lui sont fournies par

1 Inventaire $\times 1 \times 4$.

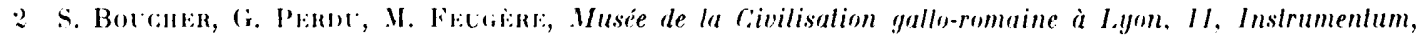

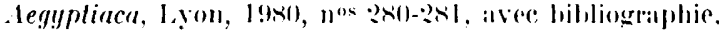

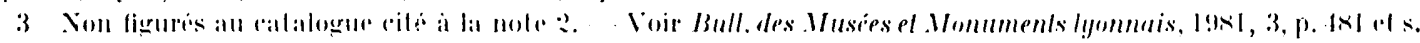

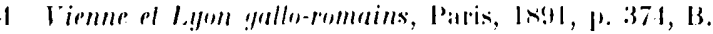

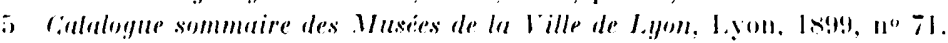

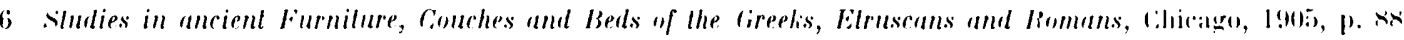
.1 101, pl. Xisll, Nix. 

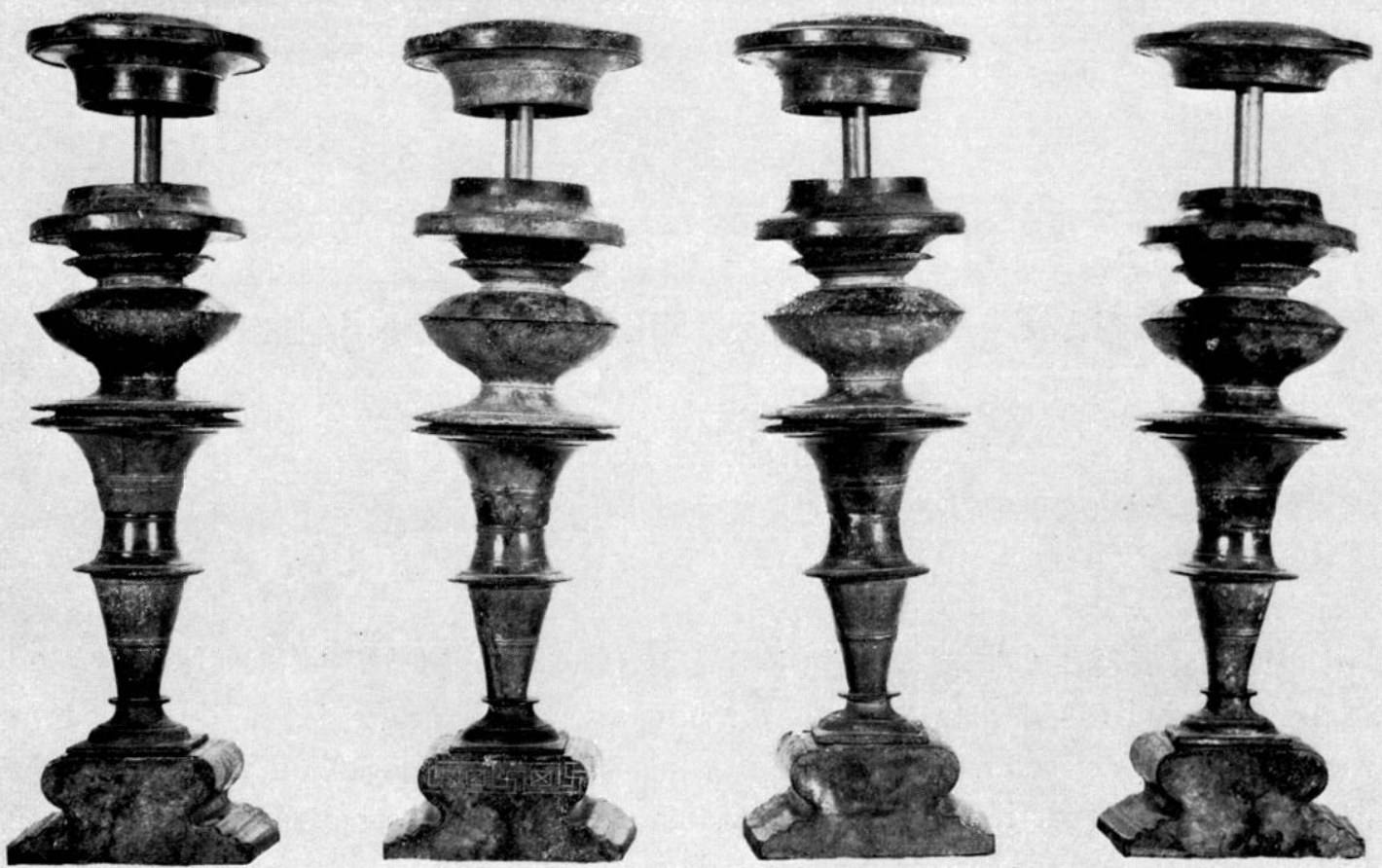

I Reronstilution provisoire des pieds de lits du .Musion de lat Civilisation gallo-romatine.
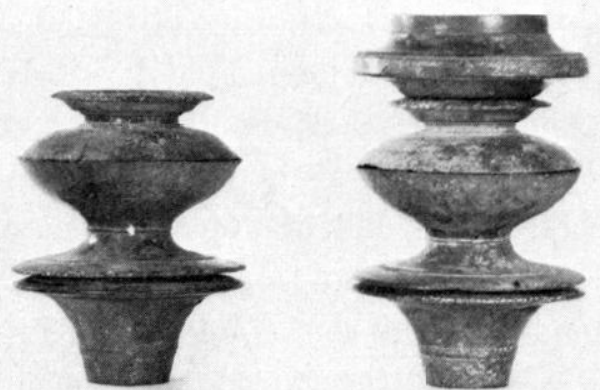

$\because$ Eklements supplimentaires de pieds de lits.

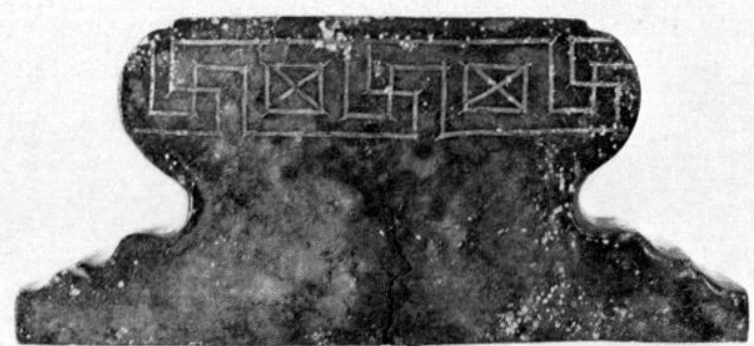

3 Pied dincortin d'incisions irh. $1: 2$. 


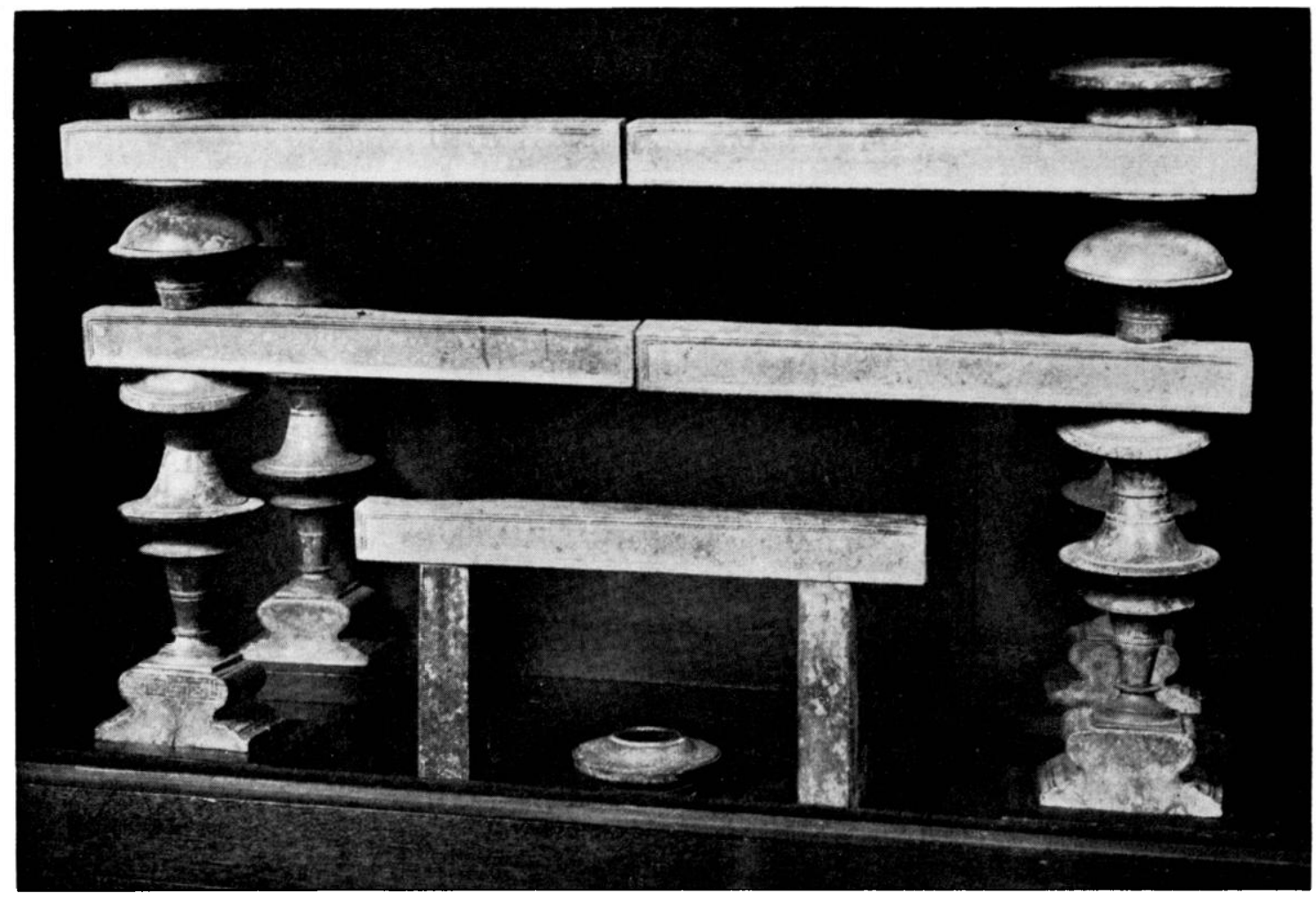

4 leconstilution des lils an bisellium dinpriss l. L. Ransom.

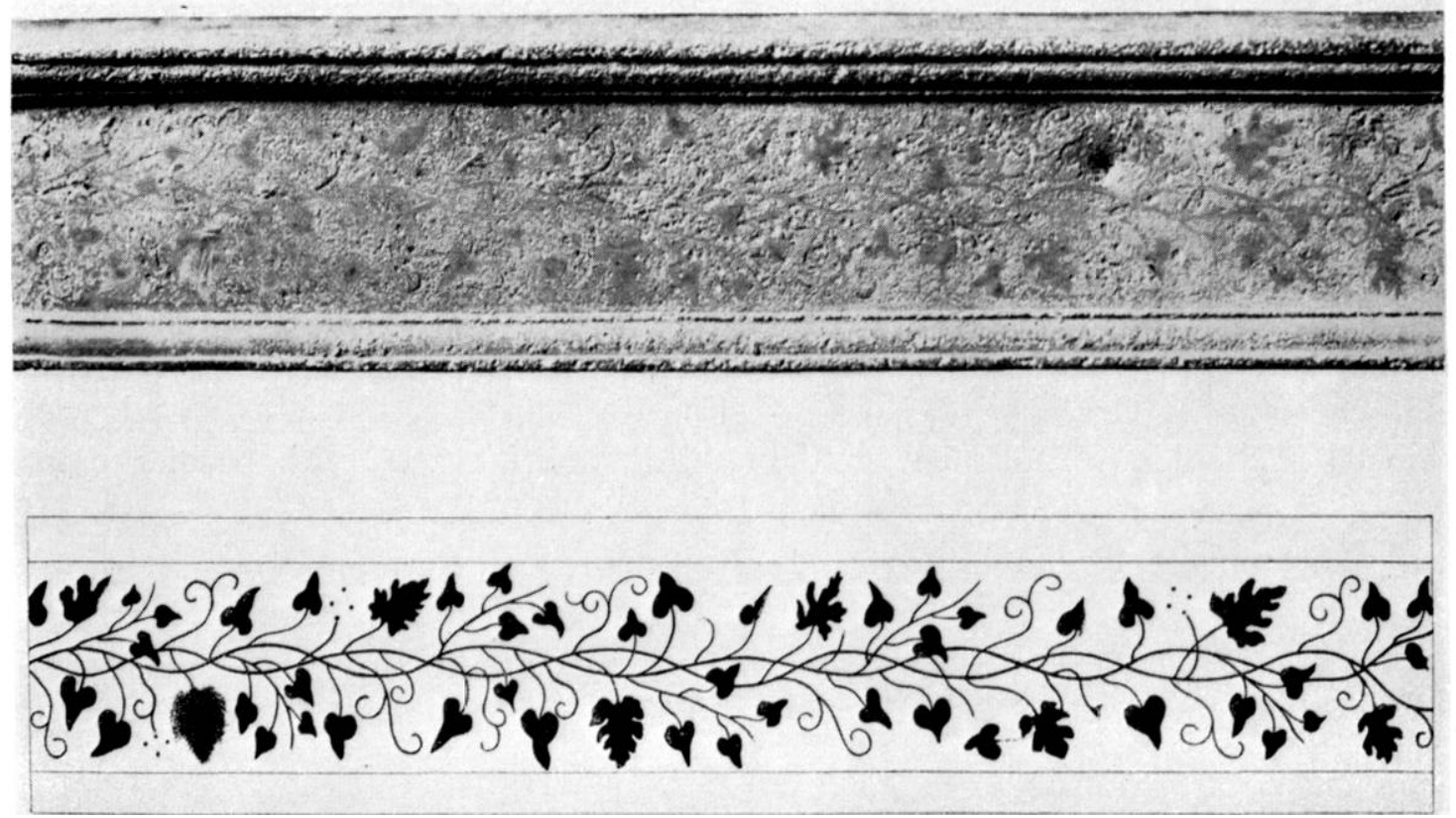

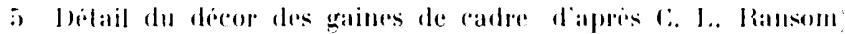


P. Dissard apparaissent cinq gaines de cadre qui, sur le cliché d'ensemble (fig. 4), semblent décorées toutes les cinq de la même facon. Un dessin de détail (fig. 亏) nous confirme la richesse de leur ornementation. Il est donc certain qu'avant 1891, ces gaines faisaient partie de l'ensemble conservé par le Musée, et justifiaient les termes "incrusté d'argent ». Il s'agit bien de la même trouvaille de Bourgoin-Jallieu, offerte au Musée par M. le duc de Luynes.

Il se trouve malheureusement que ces cinq objets ont disparu depuis longtemps. Ils ne figuraient déjà plus dans la reconstitution en lit proposée lors de l'Exposition du Bimillénaire de Lyon en $1958^{7}$. M. A. Audin qui connaît mieux que tout autre les monuments archéologiques lyonnais les plus anciens, nous a dit ne jamais avoir vu ou entendu parler de ces pièces. Le bisellium qu'a étudié C. L. Ransom a été démonté à une époque qu'il nous est impossible de préciser, ct les gaines niellées ont été égarées.

Il nous faut tenir compte toutefois du problème qu'elles posent : elles portent à quarante-sept le nombre des objets entrés au Musée en 1863. La photographie de C. L. Ransom ne nous éclaire guère, sinon qu'il y apparaît des éléments de jambages montés un peu au hasard à l'arrière du siège, et l'un d'eux, inutilisé, sous un tabouret. Il est improbable que cinq pièces complémentaires soient entrées au Musée sans qu'il y en ait la moindre trace dans les inventaires. La seule solution raisonnable est que certaines de ces pièces, soudées ou emboîtées à force, se soient séparées plus tard. Il reste curieux que ces cinq gaines de cadre, admirablement décorées, n'aient été l'objet d'aucune mention spéciale dans l'inventaire de 1863.

La trouvaille est probablement celle d'une cachette de fondeur ou de pillard, où étaient accumulées des pièces démontées. Il est étrange, lans l'un et l'autre cas, que la découverte n'ait rien offert d'autre que ces pièces de mobilier; nous verrons plus bas qu'il s'agit en fait de deux ou plusieurs meubles. S'il y arait eu tous les éléments de ces deux meubles, ou tout au moins une grande partie d'entre eux, cela ne serait pas passé inapercu. Il faut donc supposer que la phrase "les autres pièces de re meuble" signifie qu'il s'agissait de pièces différentes. M. Thibaud, propriétaire du terrain, semble-t-il, n’a pas été défavorisé. En contrepartie des gaines niellées d'argent, il reçut probablement le reste des belles pièces, les accoudoirs ou leurs décors en ronde bosse. Peut-être aussi eut-il dans ce partage des éléments qui nous manquent pour tous les pieds (voir ci-rlessous p. 183, éléments campaniformes), dont on ne sut que faire dans le meuble en question, et qui pouraient intéresser un amateur par leur forme amusante.

Le problème du bisellium. Ies différents éléments que nous possédons avaient donc été remontés, à la fin du siècle dernier, sous la forme d'un bisellium. I'autres meubles comparables. à la mème époque, avaient éte l'objet de reconstitutions semblables. Les exemples bien connus sont les bisellia du Lourre, du British Museum, ainsi que le célèbre "bisellio

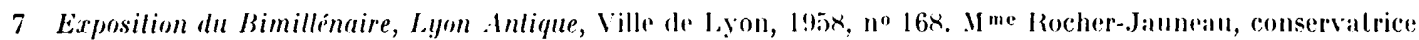

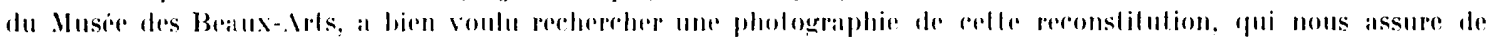
rollo allomene.

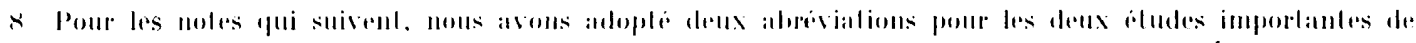

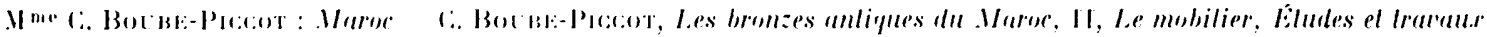

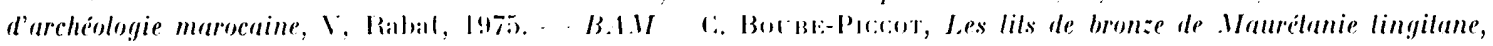

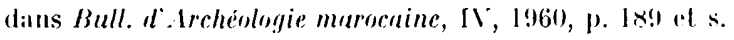

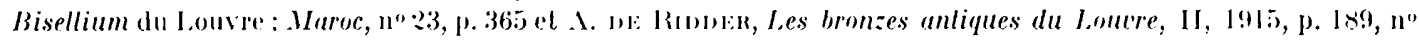




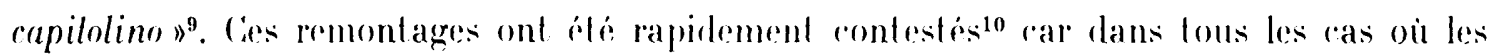
circonstances re trouraille ont ale lien étulieses, que ce soil an Asie Nincure, en Italie, et plus récemment à Mahdia of au Vlaroc ${ }^{11}$, il ne peut sagir que de lits. Nous ajouterons que ces bisellia ou fauteuils a deux places étaient, semble-t-il, très rares dans l'antiquité, et généralement accordés à titre honorifique, posl morlem ${ }^{12}$. La plupart des meubles de ce genre, d'époque hellénistique dromaine, trouvés ou non dans des tombes, ne sont pas des bisellia.

Èn ce qui concerne les pièces de bronze qui ont été données au Musée des Beaux-Arts à I.yon, la reconstitution en bisellium n'est pas satisfaisante, sur le simple plan de la logique.

Il est improbable quiun siège, à une ou deux places, ait été orné de deux gaines de cadre mises bout à bout, alors que cés gaines se présentent en réalité comme des pièces individuelles, chacune entourée d'un pourtour mouluré. Une seule gaine, plus longue, aurait été prévue. D'autre part, toutes celles que nous connaissons sont en fait des cornières, fermées à une extrémité, ouvertes à l'autre, destinées en mème temps à protéger et à décorer les angles des meubles ${ }^{13}$. L'hypothèse d'un tabouret pour utiliser la cinquième gaine est tout à fait discutable. Sur les monuments figurés, les meubles bas représentés devant les lits sont souvent de petites tables portant différents objets ${ }^{14}$. Il y a certes aussi des tabourets (subsellia) ${ }^{15}$, mais il n’en a jamais été trouré qui fussent associés de facon certaine aux trouvailles de lits au cours des fouilles ${ }^{16}$.

Nous possédons donc à Lyon cinq gaines de cadre. On conteste maintenant la nécessité d'un carlre intermédiaire entre les éléments de jambages. Chaque lit possède quatre de ces cornières (deux au moins sont décorées à l'avant). Yous avons en plus les éléments de deux pieds complémentaires. Il s'agit done ici de deux lits au moins.

Les éléments conservés. La reconstitution provisoire (fig. 1) a été effectuée en tenant compte de la progression des diamètres internes des différentes pièces, et de la logique d'une superposition qui correspond aux nécessités proposées par leurs formes, et par les remontages effectués ailleurs, dans lequels nous retrouvons les mèmes éléments ${ }^{17}$. Nous verrons

3673. Il a été depuis reconslilué en lit; mais les quatre pommeaux, hien que de dimensions comparables, portent des moulures différentes : il s'agit en fail des restes de plusieurs meubles. - Bisellium du Brilish . Huseum : . Maroc, nos 89-90, p. 376 et C. L. Raxsou, op. l, p. 98 et s., pl. VIJI-X et fig. 49. . Il existe onze pommeaux au British Museum, donc les restes d'au moins quatre lits. - Cf. aussi l'ancien bisellium de la Walters Art Gallery à Baltimore, D. KExt IIns, A Bronze Couch, dans The Journal of the Wallers Art Gallery, 1952-1953, p. 49 et s., fig. I et 2 , maintenant remonté en lit.

9 Maroc, no 102, p. 377 pt (. M. A. RicuT:R, Ancient Furniture. A IIstory of Greel, Elruscan and Roman Furniture, Oxford 1926, p. 131, fig. 311. Il a été reconstitué en lit : G. M. A. Ruchtra, The Furnilure of lhe Greehs,

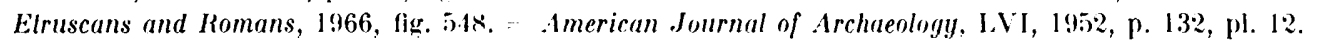

10 E. Perxice, Archänlogischer Anzeiger, 1900, p. 178 el s., a propos d'un lil de Boscoreale. C. L. Ravson,

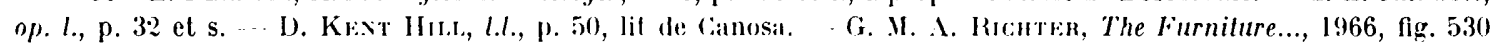
et 532, lits de Pompéi et Boscoreale.

11 Maroc, p. 359 el s., hibliographies exhaustives.

12 Cif. Dict. Int., art. Bisellium.

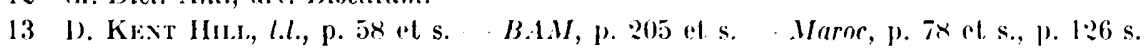

14 G. I. A. Riciten, The Furnilure, 1966, "n" 311.

$15 \quad$ Ibid., nos $311,519(?)$, p. 104 (.t s.

16 Cf. Maroc, et note suivante.

17 En particulier : Campanie, ancien bisellium du British Museum, cf. n. 8 . - Boscoreale, Pompei, cf. n. $\varepsilon$ et 10. - Amiterne, ancien bisellio capilolino, cf. n. 9. ...- Rome (?), ancien bisellium du I.ouvre, cf. n. 8 . Canosa, 
plus bas que nous devons réint roduire dans not re reconstitution theorique, entre les éléments cel $d$ ainsi que $f$ et $g$ (fig. 6), des pièes qui sont. rertainement manquantes.

Eliments (coupes, fig. 6 bis et filer):

a (lig. 3) et 7). Socle orni de moulures et d'une doucine se terminant par une gorge en piédourhe. surmontée d'un tore portant une moulure plate. L'arriere n' est pas fermé, et est plus large que la partie avant.

b) (lig. 8). Plinthe 'arrée surmontée d'un talon renversé, porlant une moulure el s'étranglant sous un cavet droit bordé d'un pelit bandeau.

c (fig. 9: Il est tronconique, orné (c I. c II) ou non $(c I I I, c I V)$ d'une moulure, el bordí d'un ressaut à sa parlie supérieure; il est lisse a sa partie inférieure qui venait, s'emboiler dans l'élément $b$.

$d$ (fig. 10): Ia partie supérieure es approximativement cylindrique: au-dessous l'élément sévase en cavel renversé. souligné d'une moulure et d'un large plateau.

$e$ (fig. 11). I'n trone de cone moulurí s'évase: en un cavet droit supportant un disque excentro épais, au-dessous d'un bandeau lisse.

$f$ (fig. 12). In large plat eaul est surmontio d'une baguelle. Au-dessus, une gorge moulure a sal part ir supérieure se rétrécil pour s"éviser ensuite en une coupole renversée.

g (fig. 13). Ine coupole plus basse que la pricédente mais de mème diamètre. est surmontée d'une gorge moulurée. puis d'un talon droit supportant une large bordure dépassinte.

$h$ (fig. 11\%. Elément supérieur du jambage. supportant le cadre du lit. Il présente un cavel droit, un listel haul. un cavet renversié el un bandean vertiral.

$i$ (fig. 15)-18:. Pommean place a l'angle du lit.

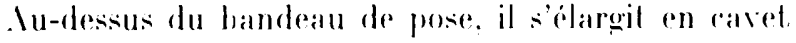

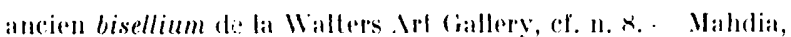

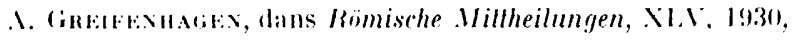

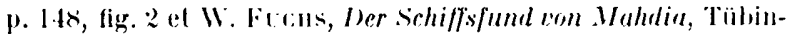
gen, 1963, p. 2x, pl. 3x; dessin dans B.1.1/, p. 263, fiy. 3, 11 .

Tamara Kspagne : L. Bato., Noltas de Arqueologia palentina, $14,19 \times 0$, p. 2.1, fig. 1. Marror, pl. 11. - B.1.\%, p. 16:3, fig. 3, ].
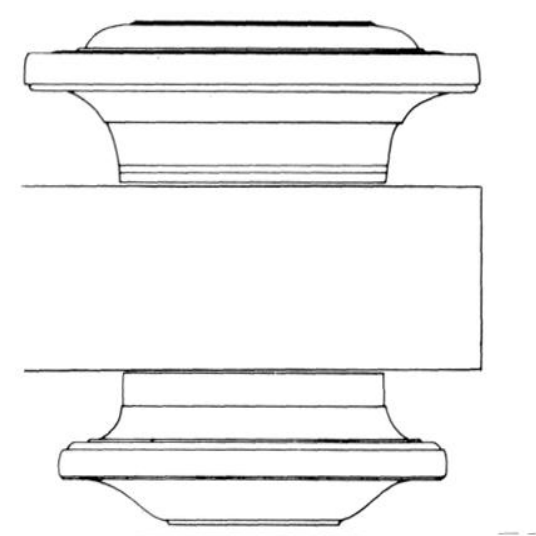

$\mathbf{h}$

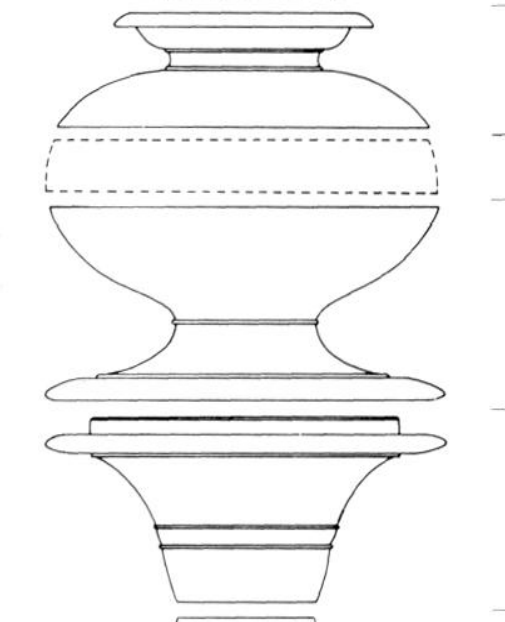

$\mathbf{g}$
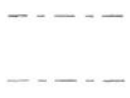

$\mathbf{f}$

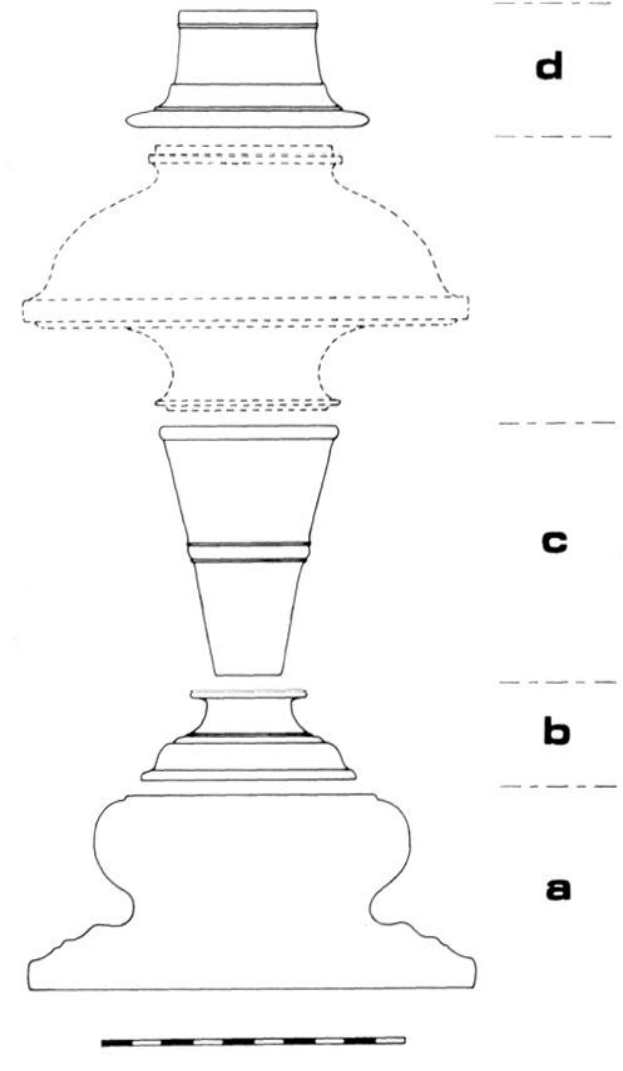

6 Montage des différents éléments. 


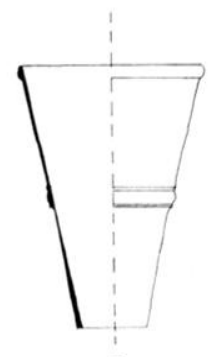

C

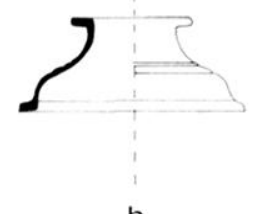

b

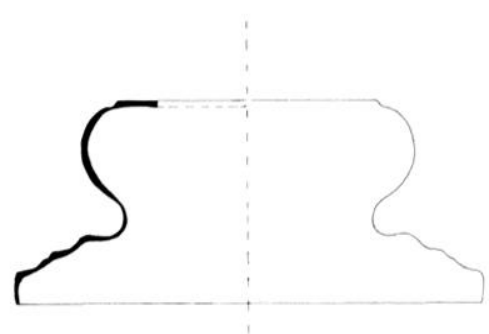

a $5 \mathrm{~cm}$
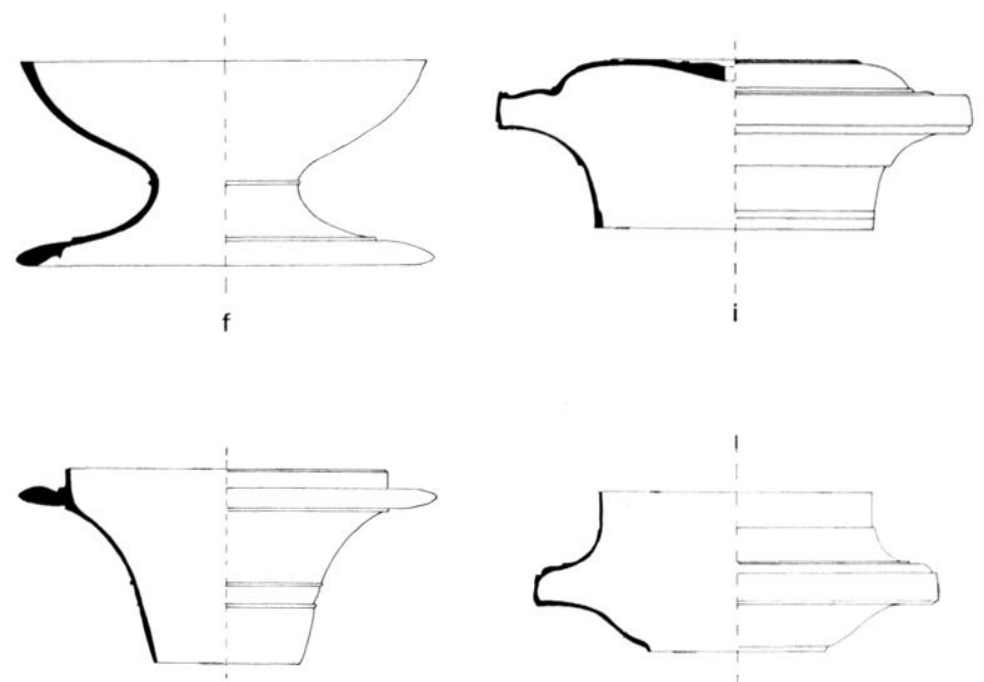

e

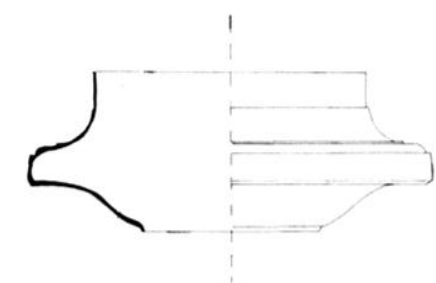

h

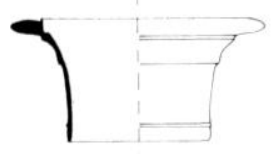

d

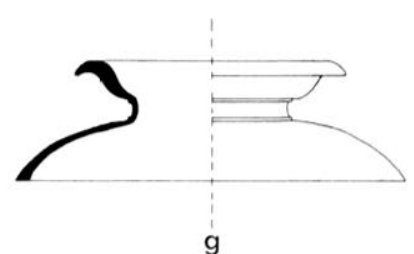

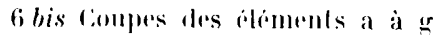

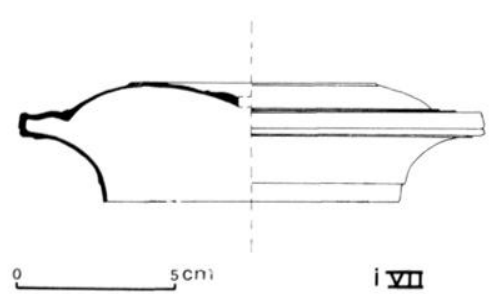

(iler rompe de l'éliment i vil

droil, surmonté d'un haul listel. puis s'arrondil en coupole surbaissée ornée de moulures. Il est pourvu en son centre d'un orifire destiné a la fixalion des arcoudoirs.

Nous noterons : les quatre pommeaux $i$ sont formés de deux pieces soudées au niveau du listel ;

le pommeau $i$ VII présente des dimensions différentes de celles des trois autres (voir le tableau). Il est aussi plus usé el détérioré. I sa part ie supérieure il est moins épais (0.3 cm) que les trois autres pommeaux (0,5 $\mathrm{cm})$;

le mème pommeau $i$ III (figr. 19) porte sur sil parlie inférieure, pris du bord, quatre encoches antiques, ainsi qu'une suite de lettres grecques inscrites en pointillé (haut. 0.3 a $0,4 \mathrm{~cm}$ ). A l'intérieur 
178

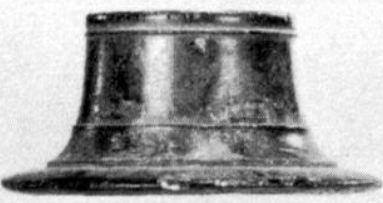

10 Filement d iech $1: 2$.

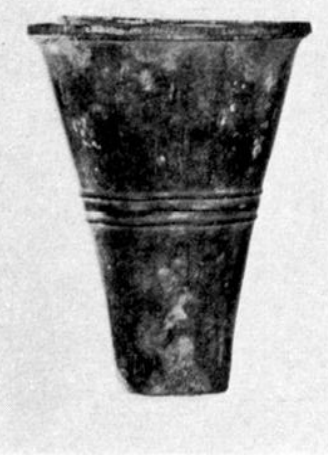

(1) Element o ich 1:2.

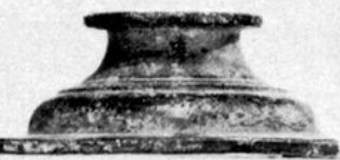

$x$ Elemenl b éch. $1 \cdot 2$.

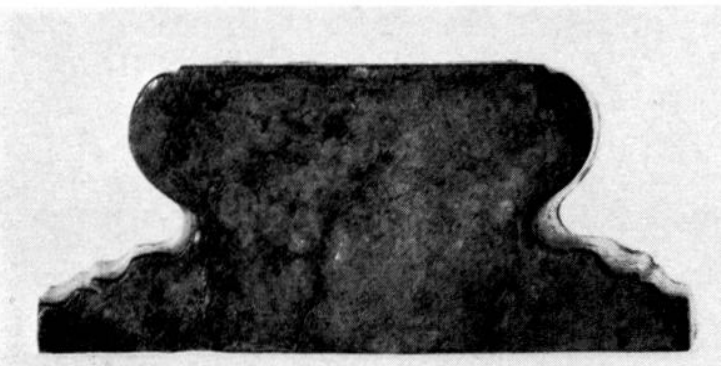

7 Filément a. Socle éch. $1: 2$.
STEPHANIE BOLCHER

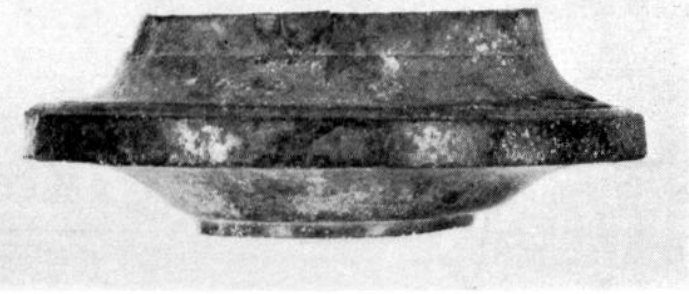

14 Element h ich. $1: \%$.

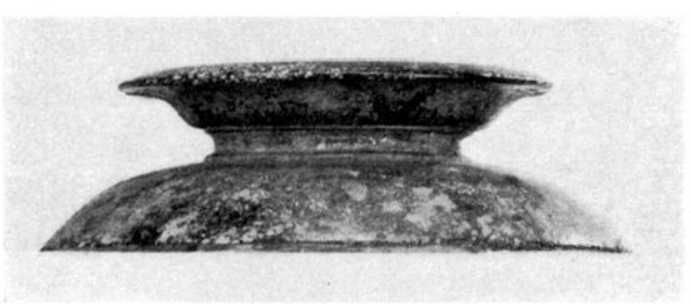

13 Ejlement g ech. $1: 2$.

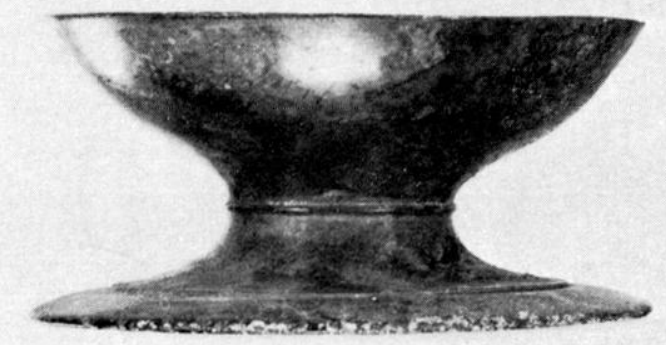

1.2 Eliment $f$ ech. $1: 2$

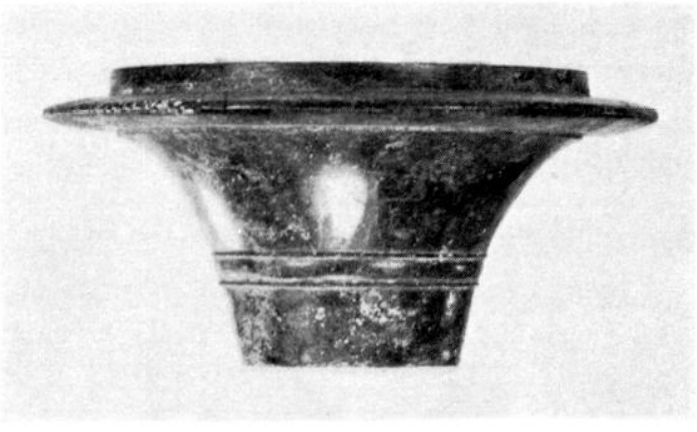

11 biment e fich. 1:2;. 

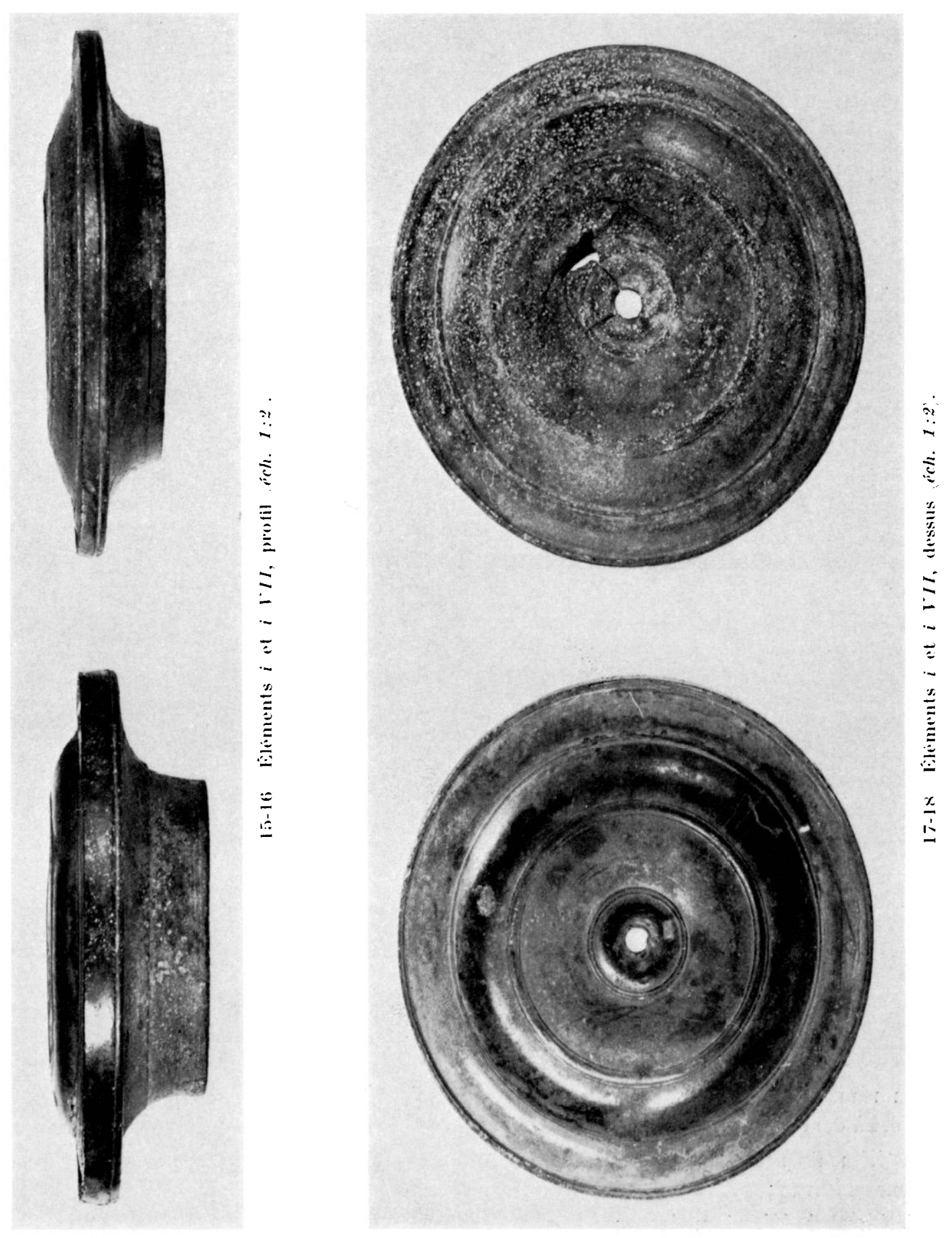


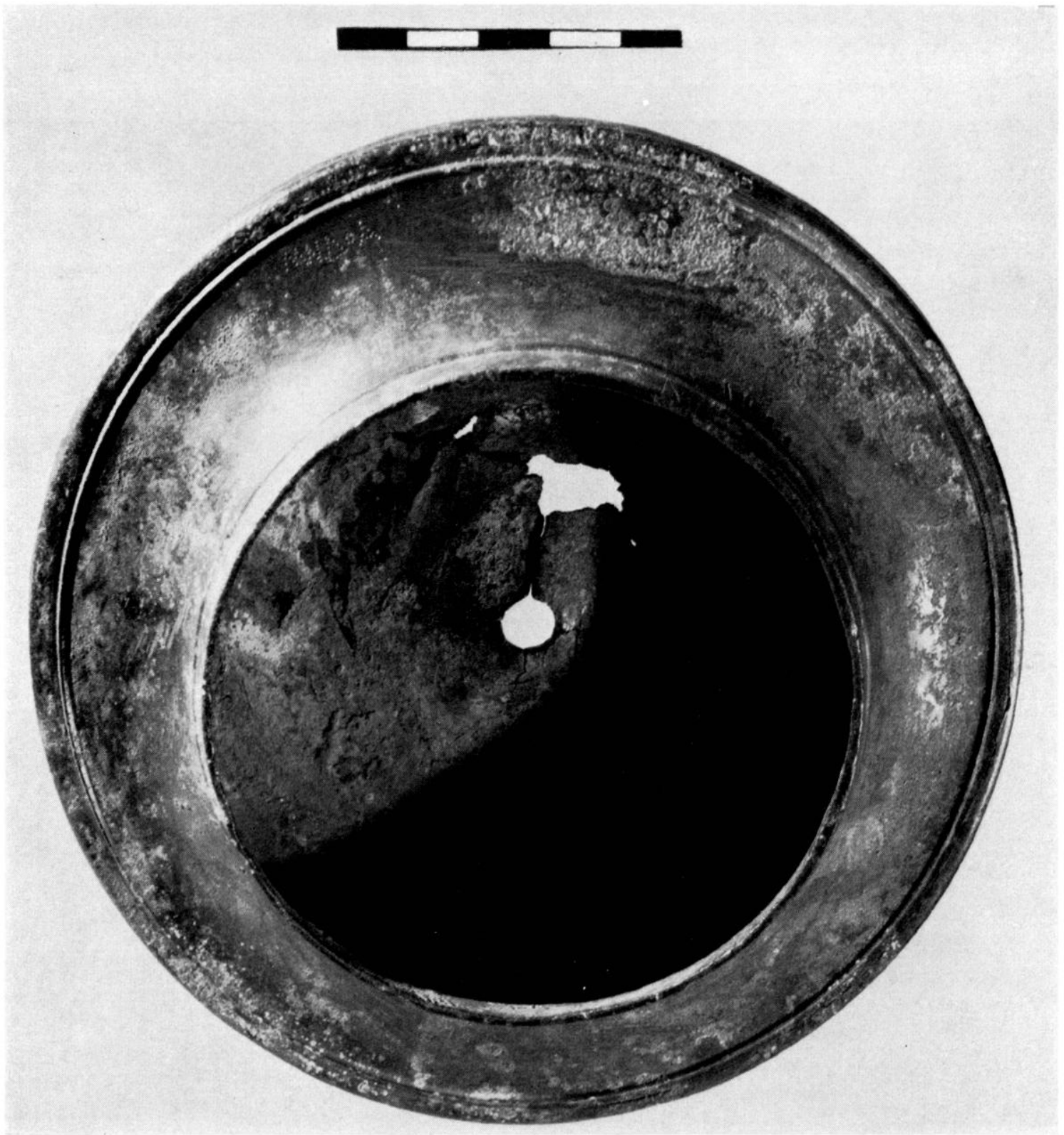

19. Jessous du pommoall $i$ VII inscriphlion ell poinlillé (éch. $1: 1$ ).

du pommeau, toujours sur cette seconde piece, apparaissent lrois lellres grecofues graves au trail. (haut. 0,4 i $0,5 \mathrm{~cm}$ ). Nous reviendrons plus bin sur ces deux inscriptions ;

les éléments $h$ sont écalement formés de deux pieces soudées, $h /$ el $h$ l possident un bandeau supérieur moderne;

l'élément bIV est moderne;

le socle a VIII porte un décor incisé sur la partie de sa fare qui correspond au tore lateral. Ce décor est constitué de grecques entrecroisese el de rectangles harrés en diagonale. Vous ne savons: pas si cette ornementation etail incrustere de melal précieux (ig. 3).

La plupart des pieces presentent des fissures el des arrachements, en particulier le pied a VIII. 
Nous observons des réparations anliques ol modernes. Cess dernières sont particulièrement importantes sur les éléments $c I, c I V, b I$.

Aucun orifice destiné à des chevilles de fixation n’apparaît sur aucune des pièces.

La fabricalion ol le mélal. Les éléments sont fails en bronze coulé, puis tourné et poli. Leur épaisseur varie d'un a plusieurs millimetres (voir les coupes. Nous noterons par exemple que les roupoles $f$ et g présentent des bords particulièrement épais, destinés à retenir entre elles un bandeau circulaire qui servait à les joindre.) (voir p. 183). Au point de charge de l'emboitement des éléments $g$ et $h$, l'épaisseur de $g$ est notable. Le centre des pommeaux, qui doivent soutenir le poids des acroudoirs, atteint plusieurs mm. Les moulures excentrées dépassent $0 . \overline{0} \mathrm{~cm}$.

Ces éléments semblent très minces, surtout les socles. Ils étaient renforcés à l'origine par un remplissage (bois ou autre matière) (voir p. 182). I)ans chaque série, ils semblent interchangeables (sauf le pommeau $i V I I$ ). Les quelques différences dans les dimensions, qui n'altèrent en rien la logique et la validité du montage, sont dues à de légers écarts entre des moules fabriqués sur une mème maquette, et aussi aux finitions. Nous avons pu faire exécuter des prélèvements sur une série de pièces représentant chaque élément, et sur le pommeau $i$ VII qui reste original. Yous donnons les résultats en Annexe, p. 192.

Les gaines de cadre. Nous ne les connaissons que par les photographies publiées par (. L. Ransom. Nous ignorons leur mode de fabrication et la technique de leur niellure. Grâce aux documents dont nous disposons, cependant, nous pouvons retrouver leurs dimensions approximatives : près de $49 \mathrm{~cm}$ de longueur, un peu plus de $4 \mathrm{~cm}$ de hauteur. Ces dimensions correspondent à celles d'un certain nombre de gaines de carlres connues par ailleurs ${ }^{18}$.

Les dessins proposés par (i. L. Ransom sont excellents. Chaque gaine possède un cadre mouluré, composé comme suit, de l'extérieur vers l'intéricur : un listel plat, un quart-derond renversé et un bandeau étroit. Le décor semble avoir été le mème sur les cinq pièces ; il comporte, d'après l'excellent dessin que nous possédons (fig. 5) deux rameaux entrelacés, l'un de lierre, l'autre de vigne. Ines tigelles issues du rameau de lierre sont terminées par un décor formé de quatre points, évoquant les baies de la plante. Le rameau de vigne présente également des tigelles, terminées par une double vrille. Ces décors sont à rapprocher de ceux de certains lits du Maroc ${ }^{19}$. Signalons le très beau décor végéral de deux gaines conservées au Metropolitan Museum ${ }^{20}$ qui ont à très peu de chose près, les mêmes dimensions que les nòtres.

Le remunlage. Nous disposons, outre les socles et les pommeaux (a el $i$ ), de sept pièces de jambage pour chaque pied. Les autres jambages connus en comportent habituellement de quatre à six ${ }^{21}$, qui sont plus complexes et plus hauts que les nôtres. Les pièces du Musée

is Maroc, p. 80, $n^{\circ} 42$. Filles peuvent itre plus ou moins longues.

19 B.1. p. 2:7. Maroc, p. $78,79,1: 26$ et s.

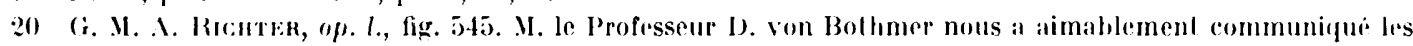
dimensions de ces gaines : long. : 49,5 cm, haut. : 4,5 cm, et nous a pricisé quelles proviennent de Perouse.

21 J. Kехт Hit., l.l., p. 53. - Maroc, p. I5. 


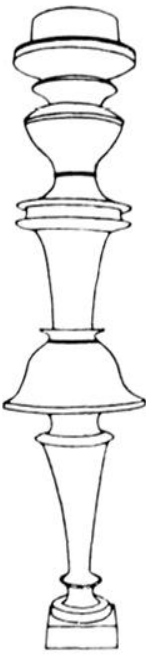

a

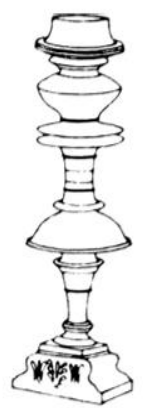

b

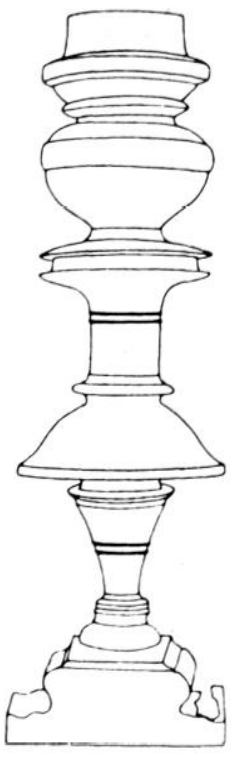

C
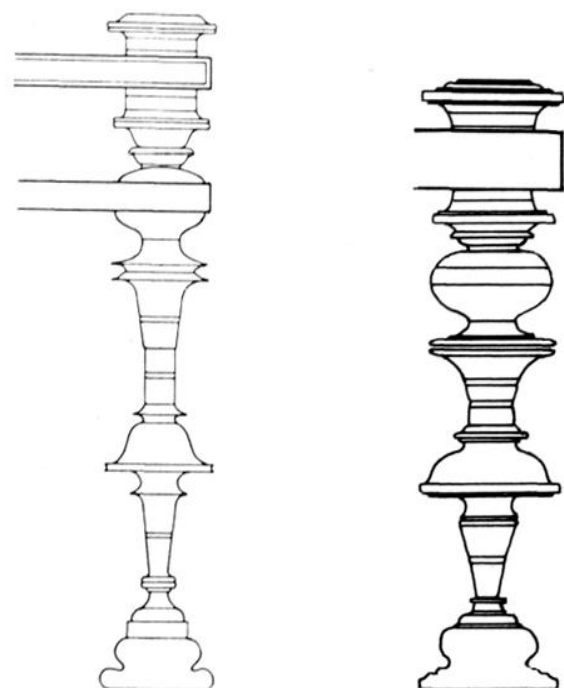

d

e

20 Silhouettes de quelques pieds de lits : a, Mahdia; b, Pompéi; c, Maroc dalpres c: Bouble-Piecot; d, lanosa (d'apres I). Kent Ilill; ; r. Bourgoin-Jallien.

de Lyon, plus nombreuses et par conséquent géométriquement plus simples étaient, sinon plus faciles à fondre, du moins plus aisément aptes a receroir des élements de remplissage nécessaires à leur stabilité.

Deux points sont à étudier :

1. Sur l'ensemble des lits présentant des jambagaes comparables aux nòtres ${ }^{22}$ apparaît entre les éléments $c$ et $d$ une pièce campaniforme. Il est certain quion doil la faire entrer dans le remontage des lits de Bourgoin-Jallieu (fig. 6i. La constance dans l'utilisation de cet. élément pour un très grand nombre de sièges antiques pose problème. A quoi servait-il? Son aspect décoratif est discutable : placé bas sur le jambage, il l'alourdit, semble-t-il sans nécessité. Nous le voyons apparaitre sur les vases à figures noires, rouges, sur les reliefs grees, étrusques ${ }^{23}$. Il est dès une haute époque lié à une base de bonnes dimensions ${ }^{24}$; mais il est associé souvent au départ à des pieds minces, fusiformes ${ }^{25}$. Nous proposons à titre d'hypothèse, qu'il put avoir une valeur fonctionnelle : relativement large, rendu pesant soit par sa propre matière, soit par un remplissage lourd, à ce tiers inférieur du jambage, il pouvait assurer l'assise et la stabilité du meuble. Il n'aurait été conservé ensuite que par habitude pour nos lits aux jambages mieux étayes, plus massifs et pourvus la plupart du

22 C.f. n. 17.

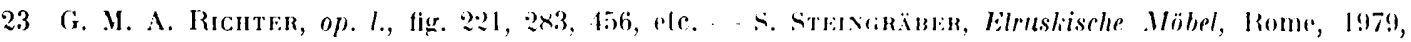
pl. XIV, XXI, XXV.

24 G. M. A. Riснт:R, op. l., fig. 221.

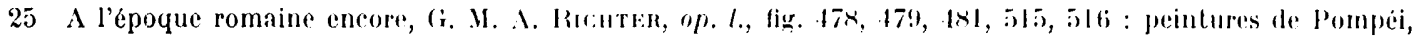
Iferculanum, Kome. 


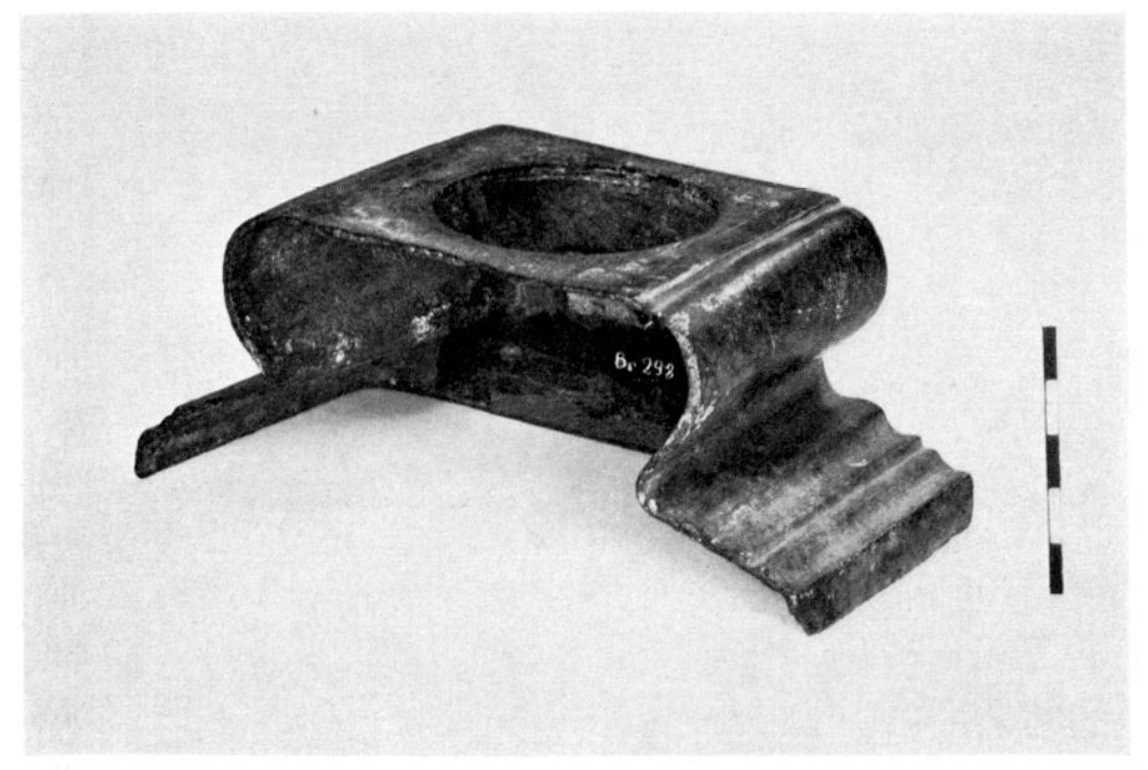

$21 \quad$ Arricrediun des socles ech. $1: 2$.

temps de bases stables, solidement assujetties par des barres transversales, ainsi que nous le verrons plus loin. Et puis avait-on peut-itte pris goùt à ces petites cloches amusantes, ou leur acrordait-on une valeur d'antiquaire. rlans une perspective où la tradition devait avoir sa part.

2. Les éléments $f$ et $g$, en forme de coupoles opposées, ne s'arlaptent pas les uns aux autres, malgré leurs dimensions très voisines. Nous remarquons que leurs bords sont relativement épais (voir les coupes, p. 177), et forment un bandeau destiné certainement à recevoir un objet de mème épaisseur. Sur la plupart des lits que nous connaissons ${ }^{26}$, une pièce intermédiaire intervient : cest une bague de bronze, de mème diamètre que les coupoles, adlérant parfois a la coupole supérieure, el présentant occasionnellement une décoration ${ }^{27}$. Dans plusieurs cas il n'en reste pas trace, el on a tenté de résoudre le problème en intercalant entre les deux coupoles un deuxième carlre de lit ${ }^{28}$. Cette hypothèse est repoussée maintenant ${ }^{29}$. Nous devons done supposer que cette bague, ou ce cerclage a simplement disparu, dans les cas où, ayant précisément une valeur ornementale, l'objet pouvait être en os, en ivoire, en bois décoré. on tout autre matière périssable.

Pour le reste, les pieds de lits do Bourgoin-Jallieu sont tres proches de ceux de certains autres lits bien connus, a quelques détails press (fig. :20) ${ }^{30}$. Les socles moulurés sont comparables en particulier à ceux des lits du IIaroc, de Pompéi, de Boscoreale, de Canosa, et même de Iélos ${ }^{31}$, a une date plus haute. Sourent ils sont déererés de motifs végétaux ou animaux

$\because 6$ 1.f. II. 17.

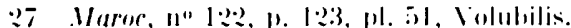

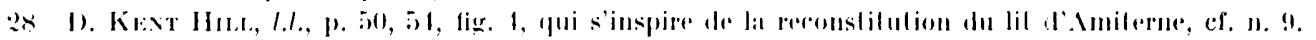

29 Maroc, p. 365.

30 Pied carre deun lit de Mahdia, differences de profil, molamment pour les élements campaniformes. 
- pattes de lion. 32. 'Trois des notres sont lisses, le quatrieme porte un simple décor incisé, sans grande valeur esthétique (fig. 3).

Nous avons signalé qu'il n'existe sur aucune des pièces conservées d'orifices destinès à la pose de chevilles de fixation. Les socles, ouverts à l'arrière, et légèrement évasés (fig. 21), recevaient, selon l'habitude ${ }^{33}$, des traverses de bois de même profil, qui assuraient la stabilité du lit. Ainsi qu'on l'a bien expliqué ailleurs ${ }^{34}$, ils étaient pourvus d'un orifice dans lequel s'emboitait une tige de bois ${ }^{35}$, sur laquelle étaient ensuite disposés les différents éléments. Au-dessus de l'élément supérieur, la tige pénétrait dans le cadre du lit, et venait s'encastrer dans le pommeau ${ }^{36}$. La stabilité du meuble était ainsi bien assurée.

Les éléments, creux et en fait très minces, ne sont que les revêtements d'un remplissage, organisé autour de la tige centrale. On a souvent retrouvé des pièces ou fragments de bois, qui, a l'origine, étaient élaborés selon le profil des revêtements de bronze, et arlhéraient étroitement à l'axe du jambage ${ }^{37}$. Yme (. Boube-Piccot avait supposé, pour un lit de Lixus, un remplissage fait en une sorte de ciment dont elle avait retrouvé les restes. Elle pense maintenant que ce sont là des noyaux provenant de la fonte ${ }^{38}$. Nous ne la suivrons pas totalement car ces noyaux sont curieusement pourvus d'un orifice central correspondant bien à une tige de montage. Il ne faut pas exclure la possibilité d'un autre remplissage que celui du bois, surtout dans le cas où les pièces sont plus complexes, comme pour le lit de ('anos ${ }^{39}$. Te plus, le remplissage do bois suppose une manufacture extrèmement complexe et coùteuse, qui se trouva peut-itre supplantée par d'autres procédés plus simples et plus efficaces.

Les accoudoirs. Les lits connus sont habiturellement surmontes d'accoudoirs qu'on appelle "fulera " ${ }^{40}$, dont il ne reste plus trace ici. Le décor de ces éléments était le plus souvent de caractère dionysiaque (protomés de mules, de cygnes, bustes de ménades, Eros, satyres). Il est probable que les lits de Bougoin-Jallieu en possédaient de cette sorte. Xous rappelons que nos gaines de cadre, ornées de pampres el de lierre, appartiennent à la mème inspiration décorative (reconstitutions, fig. 22) et 2:3).

Les dimensions. La hauteur qui peut ètre proposée iøi est évidemment théorique, puisque nous réintroduisons deux éléments dans la reconsitution des jambages. Sans

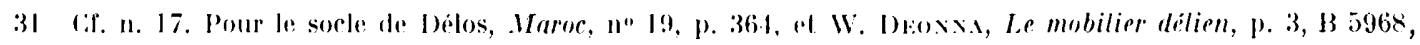
pl. IV, n" 13, qui propose une dale trop basse pour lo socle itudia.

32 A Jeelos, à Canosa, et aussi, tres recemment, pour les lits de lepave de Golfe Juan.

33 Lits de Pompeii, Boscoreale, Mahdia, n. 17.

34 Maroc, p. 15.

35) II me (.. Boube-Piecot (ibid.) a pu voir dans me maison d'llerculanum un pied dont lo montage est ainsi fait. Lous douterons seulement que laxe soit lailli dans un bois plus lendere que celui des dements.

36 la profondeur des gaines est loujours inférieure au demi-diametre du pommean; la tigre axiale l maversail donc le cadre du lit par un orifice privu à cet effet.

37 . Mares, p. 15-16.

38 B.1.Y, p. $: 217$ ol. s., puis . Maroc, p. 15,

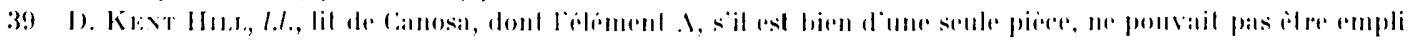
in bois.

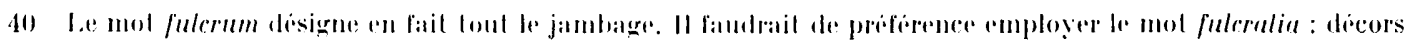
de jambage. 
LIT'S INE BOI RGOIN-JALLILE

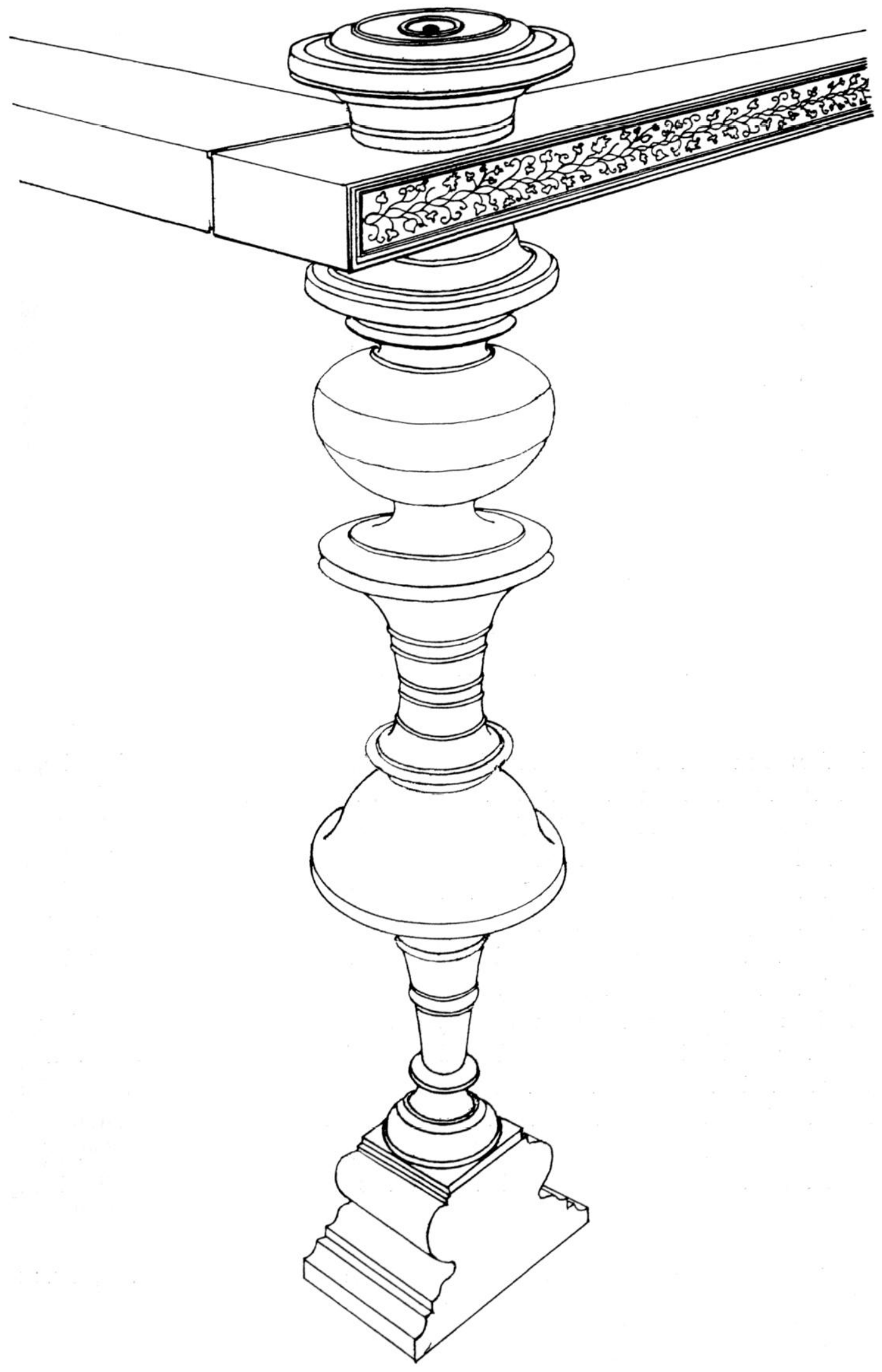

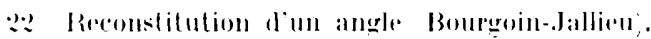




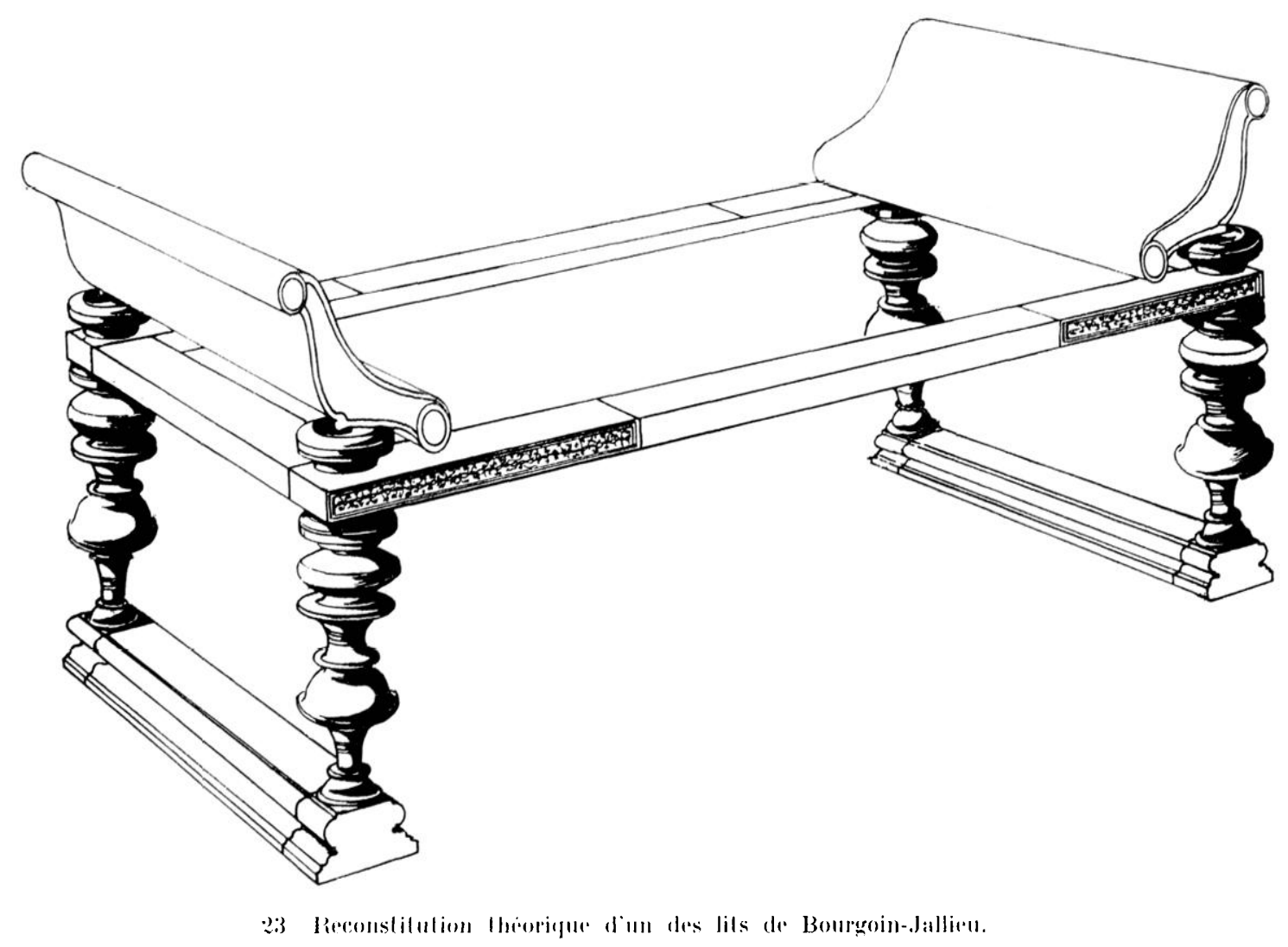

ces derniers et en prenant les pommeaux les plus hauts, ainsi que les gaines de carlee, nous atteignons environ $16 \mathrm{~cm}$. Lölément campaniforme, tel que nous pourons le supposer d'après les autres lits connus, ne devait pas excéder $7 \mathrm{~cm}$, et le cerclage entre les éléments

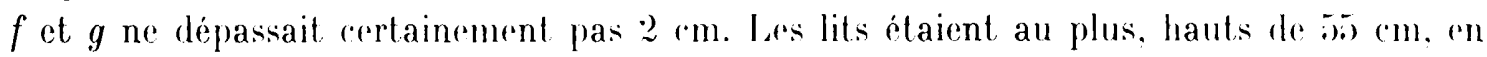
comprenant le pommean et la hase. Ce sont des lits hauts, si on les compare avee certains lits de Pompéi qui sont très bas ${ }^{41}$. Haut aussi est le lit de Canosa ${ }^{42}$. mème si l'on doit déduire quelques centimètres pour lópaisseur du carlre secondaire qui a été restitur ; il atteint 60) cm dans la reconstitution proposée.

Le diametre deséléments est important pour l'appréciation de la silhouette des pieds, fait qui interviendra dans le problème de la datation de ces meubles (ef. p. 189). Les plus grands varient de douze a quinze centimetres. Nous retrouvons ces mesures, approximativement, au Maroc ${ }^{43}$. Xous constatons au contraire que le lit de lanosa a la llalters Art Gallery ${ }^{44}$. legèmement plus haut que les nòtres. présente aussi un profil plus élancé (rliam. des pommeaux : 10 ('m). Pour les lits dont nous ne connaissons pas les mesures de facon

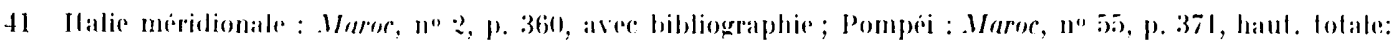

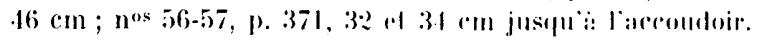

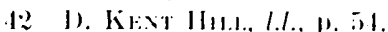

13 Maroc, p. $72,1: 010$.

44 J. Kixr Hat., ibirl., fig. 1. 
précise (Mahdia. Pompéi, Boscoreale), il nous semble, d'après les photographies et les dessins (fig. 20), que la silhouetle soit assez proche de celle des lits de Lyon ${ }^{45}$, avec un certain avantage pour ceux-ci dans le sens de la finesse.

La longueur de ces meubles reste tres théorique, puisque les cadres de bois ont disparu. On leur donne des dimensions variant de 1,75 m à $2,25 \mathrm{~m}$, en tenant compte arbitrairement des nécessités humaines. Il en est de mème pour leur largeur, tout aussi hypothétique ${ }^{46}$.

Le nombre des lits. Nous possédons certainement les restes de deux lits (fig. 1 et 2 ) : six pieds, cinq gaines de cartre, un socle décoré et trois autres lisses, deux sortes de pommeaux. Les socles sont de mèmes dimensions; on peut supposer qu'ils appartiennent aux deux lits supposés. Le quatrième pommeau, $i$ VII, pose problème : il diffère des autres, non pas tellement pour son diametre, mais pour sa hauteur, son usure, ses inscriptions, sa patine plus terne que celle des autres éléments. Il se pourrait qu il appartienne à un troisième lit dont il serait le seul vestige.

Les inscriplions. Le pommeau $i$ VII, sur sa partie inférieure, porte une inscription en pointillét7 :

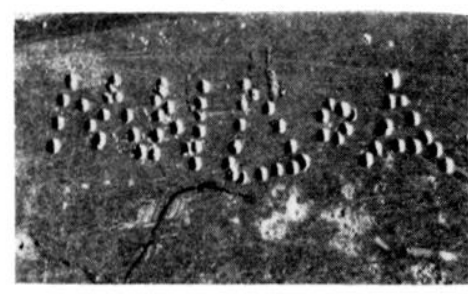

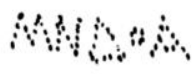

A l'intérieur du mème pommeau, toujours sur la partie inférieure, nous avons pu lire l’inscription suivante:
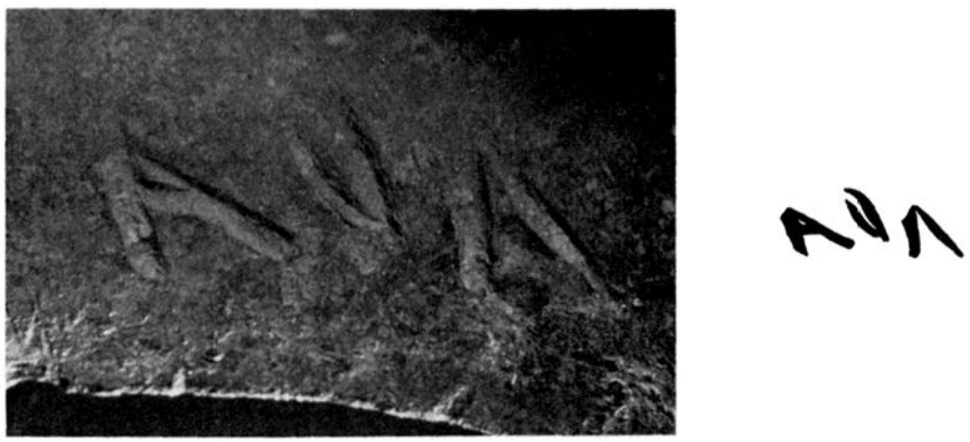

Aucune inscription n’avait été jusqürci mentionnéc sur ce type de meuble, sauf en (e) qui concerne les lits de Mahdia, pour lesquels on a parlé de lettres de montage, grecques aussi. Nous avons pu recueillir jusqu'ici les informations suivantes ${ }^{48}$ :

1) 1:f. 11. 17

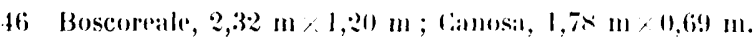

47 lecouverte par IIme .1. C.. Salvaire.

in Tous remercions lous les conservateurs el archeologues qui ont bien voulu nous aider dans notre recherche. 
Les lits du British Museum ne présentent aucune inscription ou marque*. - Le lit de Canosa au IIusée de Baltimore n'en comporte pas non plus ${ }^{50}$. - Il n'en a pas été relevé non plus sur les lits du Maroc.51. - - In lit récemment reconstitué en Espagne n'en possède pas ${ }^{52}$.

Le lit de Priène, ou plutòt quelques-uns de ses éléments, encore conservés à l'Antikenmuseum de Berlin n'en présentent pas (le lit de Boscoreale a disparu pendant la guerre) ${ }^{53}$.

Les inscriptions des lits de Mahdia n'ont pas, semble-t-il, été publiées. Nous avons pu voir récemment au Musée du Bardo deux éléments dessoudés d'un pommeau, qui portaient l'un et l'autre des consonnes grecques, gravées au trait, hautes environ d'un centimetre ${ }^{51}$. Leur suite ne propose aucun sens, et elles ne constituent certainement pas des lettres de montage.

Les lits récemment découverts à Golfe-.Juan, inédits, sont très abondamment pourvus de lettres grecques. Ces inscriptions, pour une part peut-être, seraient des numérotages ${ }^{55}$.

Sous l'un des pommeaux du meuble conservé au Louvre se trouve inscrite au trait la marque suivante, lisible dans un sens ou dans l'autre (?) : $H \vee J$. Nous n'en connaissons pas le sens.

Nous reviendrons sur la première inscription de Lyon, qui est intéressante à deux égards. D'une part, la suite MND pourrait constituer, de l'avis de M. P. Roesch, l'abréviation d'un nom, réduit à ses consonnes, tel que $M(\eta) v(i) \delta(\alpha s)$ ou $M(\varepsilon) v(\xi) \delta(\alpha \varsigma)$. A notre sens, la rosace qui suit serait un point de séparation. Quant ì la lettre A, nous pourrons proposer, a titre d'hypothèse, qu'elle soit la marque d'un atelier. Nous ne croyons pas, en tout cas, qu'elle constitue un numérotage : il s'agit de simple hon sens; les éléments des lits sont tous divers, et l'on savait très bien dans quel ordre il fallait les remonter. l)'autre part, toujours selon M. P. Roesch, et son avis est partagé par M. J. Pouilloux et par M. L. Robert ${ }^{56}$, le M et le $A$ ne peuvent itre postérieurs au ${ }_{1}{ }^{\mathrm{e}}$ siècle avant notre ère, et sont peut-être à placer assez haut dans l'époque hellénistique. Le sens de la seconde inscription AV^ (?) nous échappe.

Les dalalions. Ln des lits de Bourgoin-Jallieu datait done certainement du $\mathrm{ir}^{\mathrm{e}}$ siècle avant notre ère. Nous ne possédons aurun des accoudoirs qui entrent comme éléments

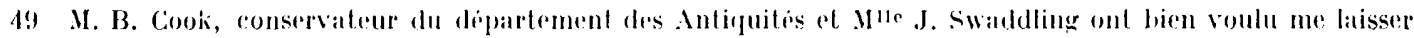
chudier tout le materiel que renferme dans ce domaine le British Musemm.

50) Tous les élements du lit de tanosa ont éte revus par Mue I). Kent IIill.

5l Confirmation m'en a été donnée par II me t:. Boube-Piccot.

52 Renseignements pris aupris de .I. A. Balil.

53) II. L: Gehrig a hien voulu faire les recherches necessaires.

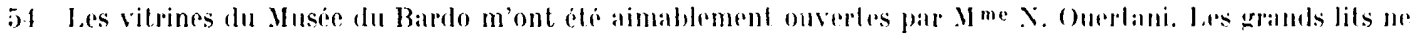
peuvent acluellement etre demontes, et je ne sais pas quelles sont les inscriplions yui ont eló vues lors des precedentes itudes.

5i) Renseiunements oblemus ampris de .I. B. I.ion.

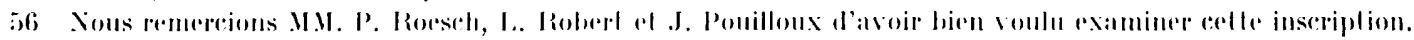

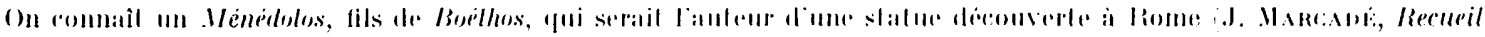

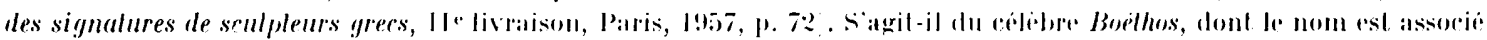

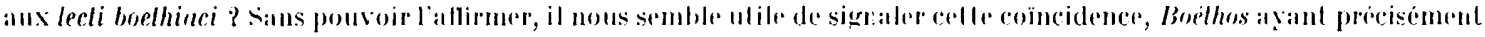

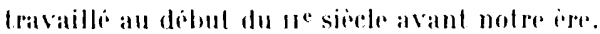


importants dans ce problème de la localisation des lits dans le temps ${ }^{57}$. Il nous reste donc les dessins des gaines de cadre, très belles, mais maintenant inexistantes, et les jambages, pour juger de l'époque à laquelle appartiennent nos lits. Le seul pommeau inscrit doit-il entrainer une datation globale pour l'ensemble de la trouvaille?

II me C. Boube-Piccot s'est occupée très récemment de ces problèmes. Les conclusions actuelles sont les suivantes : on assisterait, au cours des siècles, à un tassement, à un épaississement des sillıuettes des jambages ${ }^{58}$. Il est vrai que les lits les plus anciens ont une structure légère, comparable à celle qui apparait sur les monuments figurés. Cies lits ont été découverts sur des sites antérieurs à l’époque romaine (Phanagoric, Priène par exemple ${ }^{59}$ ). Le lit de Canosa ${ }^{60}$ bien que présentant exactement les mèmes éléments que ceux du Musée de Lyon, possède des jambages beaucoup plus minces (diamètres de $10 \mathrm{~cm}$ pour Canosa, $13 \mathrm{~cm}$ pour Lyon). Mlle I). Kent Hill, en tenant compte aussi du décor des accoudoirs, le situe au moins au début du $1^{\text {er }}$ siècle de notre èrét. Les garnitures d'accoudoirs des lits de Mahdia sont du II $^{\mathrm{e}}$ siècle avant notre ère, plus précisément du dernier tiers de ce siècle. Cependant, la silhouette des pieds de Mahdia ne diffère guère de celle de nos lits, qui nous semblent aussi plus légers que ceux du Maroc, situés par Mme Boube, pour les plus anciens, au début de l'époque romaine ${ }^{62}$; ils sont plus élancés, aussi que ceux de certains meubles de Pompéi (fig. :2(0).

Il faut donc revoir, dans une perspective très ouverte, le problème de ces meubles, où qu'ils soient trouvés, inscrits ou non inscrits, représentant un état actuel au moment de leur enfouissement, mais aussi toute une tradition, peut-ître répétitive, peut-être due au goût d'antiquaire que possédaient les Romains.

Les ateliers. Les marques grecques - - quelles qu'elles soient - relevées sur les lits mentionnés plus haut portent à croire que ces meubles provenaient d'ateliers pré-romains, sans doute d'Asie mineure, de Cirèce ou d'Italie méridionale ${ }^{63}$. Il reste probable que ce type de meuble se perpétua fort longtemps et que des ateliers d'époque romaine purent en garder la tradition ${ }^{64}$. Nous noterons qu'aucune marque laline n’a encore été relevée sur aucun d'entre rux.

Les lits de Bourgoin-Jallieu ont été trouvés hors-contexle ce qui ne facilite pas le problème. Il est possible que, comme dans les villes détruites par le Vésuve, et dans celles du Maroc, ils aient fait partie du mobilier de riches demeures romaines. Leurs dates ne sont

57 I.A.M, p. 264 et s.

is Ibid., p. 262 et s.

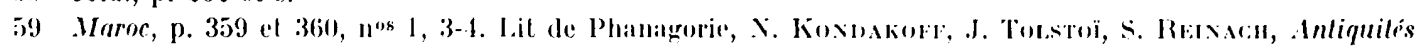

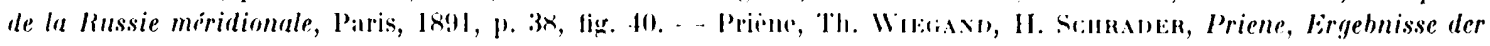

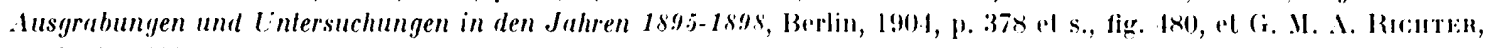
op. l., fig. 308.

60) I). Ki:x HII.I., fig. 4.

6) Ibill, 1). 61 .

(i:) Warwc, p.:2.

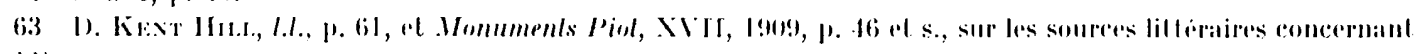
('e) problime.

64 Il me C. Boube-Piccot pense qu'il exista des ateliers au Maroc pour fabriquer ces lits. 
pas un obstacle à cette supposition. Les Romains qui s'installèrent en Gaule amenèrent certainement dans leurs bagages leurs objets personnels, fussent-ils de ces dimensions : nous avons vu qu'ils étaient faciles à monter, donc faciles à démonter. Mais nous devons tenir compte des découvertes récentes de Golfe-Juan : l'épave est certainement antérieure à la conquête romaine. Des lits tels que les nòtres ont pu être importés à la demande des habitants de la Narbonnaise, ou même des Allobroges, des Arvernes, dont la richesse était légendaire ${ }^{65}$.

Quel était leur usage? Nous ne pensons pas que ces meubles aient été des lits de triclinium, au sens antique de ce mot : ils ne sont absolument pas faits pour supporter, au cours d'un repas, le poids de trois personnes. Nous croirions plus volontiers qu'il s'agit là de lits d'apparat ou de repos ${ }^{66}$. Nous pensons en outre que leurs fonctions devaient ètre tout à fait diverses. Nous devons noter la taille très réduite des lits du British Museumb ${ }^{67}$. Ceux du Louvre non plus ne sont pas conformes ${ }^{68}$ aux exigences d'une utilisation commune. Ciertaines pièces, au Maroc, sont aussi nettement plus petites que d'autres, ainsi qu'à Pompéi69. Il se pourrait que ces meubles n'aient de valeur que luxueuse, et que les plus réduits aient été destinés à des usages autres qu'humains. Nous pensons aux lits de culte utilisés pendant les lectislernes, et sur lesquels on déposait des mannequins ${ }^{70}$.

Quant aux décors dionysiaques dont ils sont pourvus la plupart du temps, leur sens n'est pas bien établi. Il est improbable que tous ces lits aient été associés à des cultes bacchiques. La part de l'habitude dut jouer un grand ròle dans la répétition d'ornements qui s'adaptaient particulièrement bien à la forme des accoudoirs (protomés de mules, cols de cygnes, bustes de ménades et d'amours). Leur nier toute valeur cultuelle serait cependant une erreur, dans la mesure où nous leur attribuons une signification autre que simplement utilitaire. Leur présence en Ciaule, si elle est antéricure à la conquête, ne laisse pas d'être ambiguë.

\section{Stéphanie Borchtr}

(C.N.R.S.)

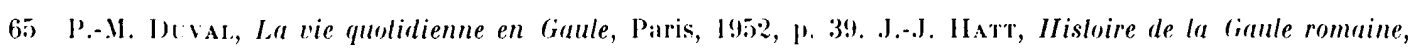

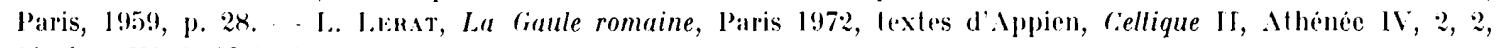
strabon IV, 1, 13 : : 188 :

66 Cif. les lits découverts dans le lablinum d'une villa pompéienne, Maroc, no 56, p. 371.

67 Nous n'avons pas leur hauleur exacte puisqu'ils sont démontis, mais ils atteignent au plus $30 \mathrm{~cm}$, dimension a rapprocher de celle des lits de lablinum de Pompeii, cf. n. 66. Les pommeitux ont 6,5 $\mathrm{cm}$ de diametre.

6x Ine grande partie des pieces de jambiges sont modernes; nous ne pouvons pas donner de hauteur exacte. Les pommeaux ont un diamitre fui varie de $9 \mathrm{~cm}$ a $9,6 \mathrm{~cm}$. Nous avous dit plus haut $(\mathrm{n}$. 8 ) qu'ils appartenaient a des lits diffierents.

69 Maroc, $\mathrm{n}^{\circ} 1333$, p. 126, sixieme éloment de jambage, diam. : $8,5 \mathrm{~cm} ;$ passim, autres déments variant de 10 à $13 \mathrm{~cm}$ de diamètre. Pompéi, cf. n. 66.

70 I)ict. Anl., art. Leclisternium.

V.B. Les coupes sont dues à Marie-Claude Salvaire fig. 24 et 25 , les pholographies à lrancois layge fig. 1 à 21 , les dessins a dain Cimal (fig. 22: 21.23 ). 


\begin{tabular}{|c|c|c|c|c|c|c|c|c|c|}
\hline & Dimensions en centimètres & I & II & III & IV & $\mathrm{V}$ & VI & VII & VIII \\
\hline$i$ & 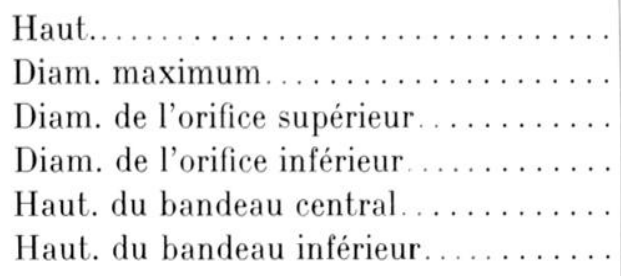 & $\begin{array}{r}4,8 \\
14,7 \\
0,6 \\
8,5 \\
1 \\
2\end{array}$ & $\begin{array}{r}4,8 \\
14,7 \\
0,6 \\
8,6 \\
1,1 \\
1,9\end{array}$ & $\begin{array}{r}4,8 \\
14,7 \\
0,7 \\
8,6 \\
1 \\
1,9\end{array}$ & & & & $\begin{array}{r}3,3 \\
14,5 \\
0,8 \\
9,3 \\
0,7 \\
0,4\end{array}$ & \\
\hline$h$ & 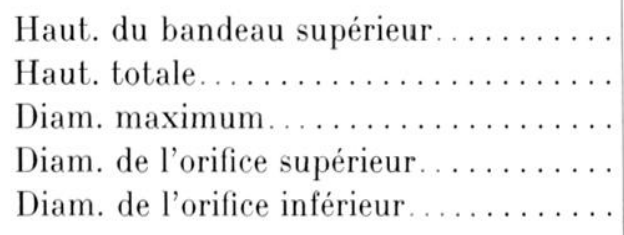 & $\begin{array}{r}(1) \\
4,4 \\
13,2 \\
8,5 \\
5,7\end{array}$ & $\begin{array}{r}1 \\
4,5 \\
13,2 \\
8,5 \\
5,8\end{array}$ & $\begin{array}{r}1 \\
4,1 \\
13,2 \\
8,5 \\
5,8\end{array}$ & $\begin{array}{r}1 \\
4,5 \\
13,2 \\
8,4 \\
5,9\end{array}$ & \begin{tabular}{r|} 
\\
1 \\
4,2 \\
13,2 \\
8,5 \\
5,9
\end{tabular} & & & \\
\hline$g$ & 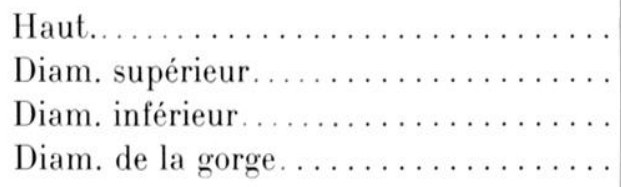 & $\begin{array}{c}3,2 \\
9 \\
12,2 \\
5,4\end{array}$ & $\begin{array}{r}3,2 \\
9,2 \\
12,3 \\
5,3\end{array}$ & $\begin{array}{r}3,5 \\
8,9 \\
12,2 \\
5,3\end{array}$ & $\begin{array}{r}3,3 \\
9,1 \\
12,3 \\
5,3\end{array}$ & $\begin{array}{r}3,5 \\
9,4 \\
12,4 \\
5,2\end{array}$ & $\begin{array}{c}3,4 \\
9 \\
12,3 \\
5,3\end{array}$ & & \\
\hline$f$ & 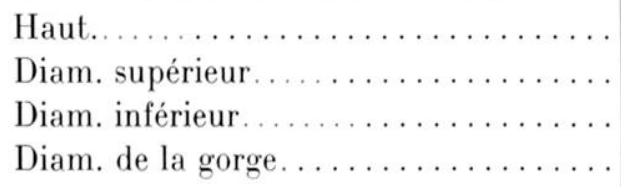 & $\begin{array}{c}5,7 \\
12,7 \\
13 \\
4,8\end{array}$ & $\begin{array}{c}5,7 \\
12,6 \\
13 \\
4,8\end{array}$ & $\begin{array}{c}5,7 \\
12,6 \\
13 \\
4,8\end{array}$ & $\begin{array}{c}5,6 \\
12,5 \\
13 \\
4,8\end{array}$ & $\begin{array}{c}5,7 \\
12,4 \\
13 \\
4,7\end{array}$ & $\begin{array}{c}5,9 \\
12,5 \\
13 \\
4,8\end{array}$ & & \\
\hline e & 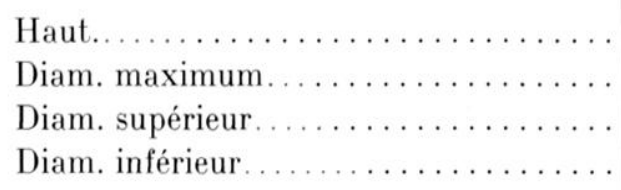 & $\begin{array}{l}5,8 \\
13 \\
10 \\
4,5\end{array}$ & $\begin{array}{l}5,9 \\
13 \\
10 \\
4,5\end{array}$ & $\begin{array}{r}5,7 \\
12,9 \\
10,1 \\
4,5\end{array}$ & $\begin{array}{r}6 \\
12,9 \\
10,1 \\
4,4\end{array}$ & $\begin{array}{c}6 \\
13 \\
9,9 \\
4,4\end{array}$ & $\begin{array}{l}6,1 \\
13 \\
10,1 \\
4,5\end{array}$ & & \\
\hline$d$ & 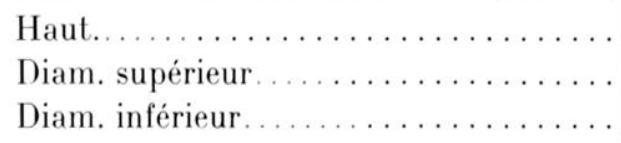 & $\begin{array}{l}3,4 \\
4,3 \\
8\end{array}$ & $\begin{array}{l}3,6 \\
4,3 \\
8\end{array}$ & $\begin{array}{l}3,4 \\
4,3 \\
7,8\end{array}$ & $\begin{array}{l}3,2 \\
4,5 \\
7,9\end{array}$ & & & & \\
\hline$c$ & 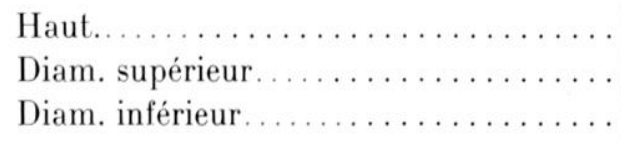 & $\begin{array}{l}7,6 \\
5,8 \\
2,2\end{array}$ & $\begin{array}{l}7 \\
5,9 \\
2,2\end{array}$ & $\begin{array}{l}7,5 \\
5,9 \\
2,4\end{array}$ & $\begin{array}{l}7 \\
5,9 \\
2,3\end{array}$ & & & & \\
\hline$b$ & 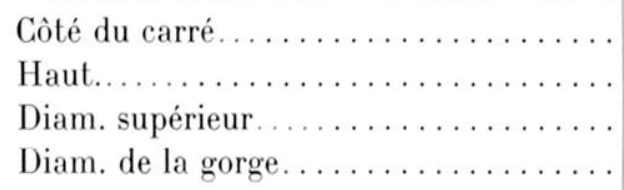 & $\begin{array}{l}7 \\
2,7 \\
3,7 \\
2,9\end{array}$ & $\begin{array}{l}7 \\
2,6 \\
3,9 \\
2,8\end{array}$ & $\begin{array}{l}7 \\
2,6 \\
3,9 \\
2,8\end{array}$ & $\left.\begin{array}{l}{[7} \\
{[2,7]} \\
{[3,7]} \\
2,9]\end{array}\right]$ & & & & \\
\hline$a$ & 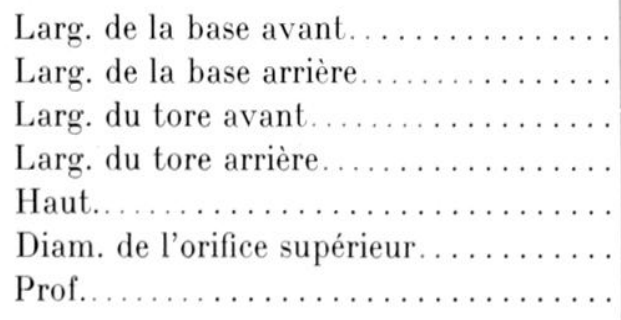 & $\begin{array}{c}14,4 \\
15,5 \\
10,3 \\
11,2 \\
6 \\
5,9 \\
8,3\end{array}$ & $\begin{array}{c}14,2 \\
15,3 \\
10,2 \\
11,1 \\
6 \\
5,9 \\
8,4\end{array}$ & $\begin{array}{l}14,6 \\
15,4 \\
10,4 \\
11 \\
5,9 \\
5,7 \\
8,5\end{array}$ & & & & & $\begin{array}{r}14,5 \\
15,5 \\
10,3 \\
11,2 \\
5,9 \\
5,6 \\
8,4\end{array}$ \\
\hline
\end{tabular}


INNFXE

Analyses

Cuivre Etain Plomb Zinc Antimoine

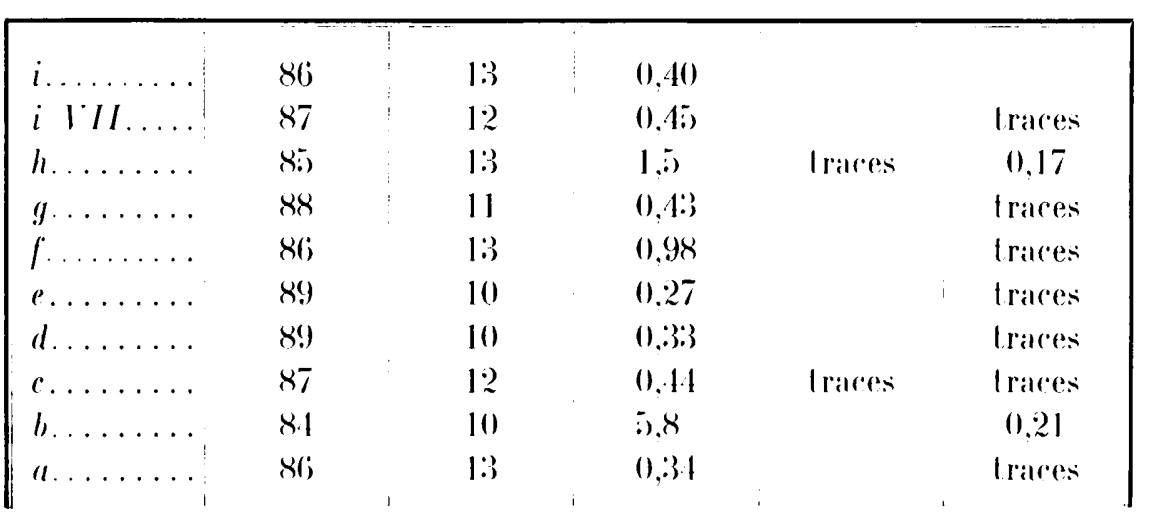

Les analyses ont été effectuées au Laboratoire de spertrographie de l'Institut de Chimie et de Physique Industrielles (ICPI) des Facultés calholiques de lyon, sous la direction de $\mathrm{M}^{\mathrm{me}} \mathrm{F}$. Formenti. Le cuivre est calculé par différence, en tenant compte d'un taux global maximum d'impurelés. Les éléments majeurs sont donnés avec deux significatifs à l'unilé la plus proche.

Les échantillons ont été prélevés sur une série complète d'éléments de a à $i$. Le pommeau $i$ I'lI qui se distingue des trois autres par ses inscriptions el ses dimensions a fail l'objet d'une élude supplémentaire.

Yous pouvons faire les remarques suivantes : les taux de cuirre sont très réguliers, de 86 a $89 \%$. . Les taux d'élain, de 10 a $13 \%$ restent dans la moyenne de ceux qui ont été relevés pour l'analyse des objets de statuaire ${ }^{71}$. Les taux de plomb sont tres faibles. Dans huit cas sur dix ils sont inférieurs à $1 \%$ : l'analyse de l'élément $h$ en présente $1,5 \%$. Il est probable que le prélévement a concerné un endroit où s'était formé, par hétérogénéité, un grlobule de plomb ${ }^{72}$. - L'élément $b$ comporte $5,8 \%$ de plomb, et se distingue nettement en cela de la composition des autres éléments. Cette pièce est en mauvais état, mais nous avons vérifié qu'il n'y avait aucune trace de soudure au plomb à l'endroit du prélèvement. Nous pensons que, perdue ou brisée, elle avait déjà été remplacée dans l'antiquité, et que pour cette réfection, on a utilisé un bronze courant, plus riche en plomb.

Ces résultats sont très cohérents. Yotons que concernant des éléments pris au hasard, puisqu'ils sont interchangeables, ils nous donnent une image certainement exacte de la composition de l'ensemble des pièces. Ils proposent un type de bronze nouveau. pratiquement dépourvu de plomb.

Nous notons que les deux pommeaux analysés, de séries certainement différentes, ont des compositions très voisines, ce qui peut porter à les considérer comme étant soit d'une même époque, soit d'un mème atelier, soit correspondant à une formule particulière à ce genre d'objet. kin effet,

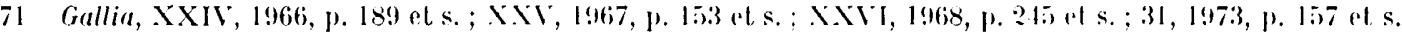
72 Giallia, XXIV, 1966, p. 198. 
nous considérerons l'analyse effectue pour le lit de Cimosa (voir plus haut, p. 175, n. 8 et 17), qui présente pris de $86 \%$ de cuive, pris de $11 \%$ d'étain, et $0,0 \%$ de plomb. Celte lechnique est-elle liée a l'époque hellénistique, ou bien ful-elle employée également à l'époque romaine? L'analyse des éléments des pieds de lits du Maroc pourra apporter quelques éclaircissements à ce sujet. Cette qualité de bronze pauvre en plomb sc justific pcut-être par la fonction de ces pièces de lit : mèmes renforcées par des portants et des remplissages (roir ci-dessus, p. 181), elles devaient exiger une certaine rigidité.

Nous avons pensé à une parenté de cette technique avec celle de la vaisselle, tout au moins pour certaines pièces qui sont fondues et tournées, et dont les formes, par les courbures et les moulures, se rapprochent des nòtres. Mais nous constatons que la composition de ces objets, en particulier des casseroles et des patères de bain, que nous avons plus particulièrement étudiées ${ }^{73}$, propose des taux de plomb extrèmement variables : de $0 \%$ a :30\%. Ces résultats sont confirmés par l'analyse de la vaisselle effectuée par M. II. P. den Boesterd et E. Hockstra ${ }^{74}$ où l'alliage des casseroles et patères de bain, sans aller aussi loin, peut présenter jusqu'à $12 \%$ de plomb, qu'il s'agisse de l'anse ou du récipient lui-mème. Toutefois les listes proposées dans l'ouvrage cité montrent que le plomb est le plus souvent absent pour nombre de pieres de la raisselle antique. D'autres analyses sont nécessaires, qui pourraient ètre, dans notre cas précis, un élément de datation.

\section{F. Formwtis el S. Botchis}

73 Fiallia, XXIV, 1966, р. 212; XXV; 1967, p. 2:99.

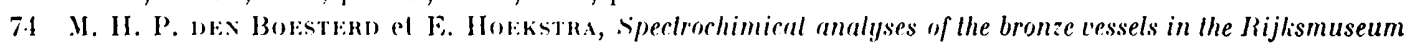
G. M. Kam al Nijme.jen, I.eyde, 1966. 